JOURNAL OF

FUNCTION SPACES AND APPLICATIONS

Volume 4, Number 3 (2006), 275-304
(C) 2006, Scientific Horizon http://www.jfsa.net

\title{
New classes of rearrangement-invariant spaces appearing in extreme cases of weak interpolation
}

\author{
Evgeniy Pustylnik and Teresa Signes* \\ (Communicated by Fernando Cobos)
}

2000 Mathematics Subject Classification. Primary 46B70, 46E30; Secondary 46E35, 46M35.

Keywords and phrases. Ultrasymmetric spaces, weak type interpolation.

\footnotetext{
Abstract. We study weak type interpolation for ultrasymmetric spaces $L_{\phi, E}$, i.e., having the norm $\left\|\phi(t) f^{*}(t)\right\|_{\widetilde{E}}$, where $\phi(t)$ is any quasiconcave function and $\widetilde{E}$ is arbitrary rearrangement-invariant space with respect to the measure $d t / t$. When spaces $L_{\phi, E}$ are not "too close" to the endpoint spaces of interpolation (in the sense of Boyd), the optimal interpolation theorem was stated in [13]. The case of "too close" spaces was studied in [15] with results which are optimal, but only among ultrasymmetric spaces. In this paper we find better interpolation results, involving new types of rearrangement-invariant spaces, $A_{\phi, \mathbf{b}, E}$ and $B_{\phi, \mathbf{b}, E}$, which are described and investigated in detail.
}

* Supported in part by Ministerio de Educación y Ciencia MTM2004-01888 (Spain) and by Fundación Séneca PB/21/FS/02 (CARM). 


\section{Introduction}

Weak interpolation appeared in [5] as generalization of the famous Marcinkiewicz theorem. In [10] (Section 6 of Chapter II) and in [4] (Section 7 of Chapter 4) this kind of interpolation was extended to couples of spaces with arbitrary fundamental functions. Various powerful applications of this theory to differential and integral equations, Fourier series and transformation, Sobolev embeddings etc. were due to its main peculiarity: the strong properties of (quasi)linear operators in intermediate spaces can be obtained under rather weak conditions at endpoint spaces. This was particularly important for integral operators, satisfying only such conditions in spaces like $L_{1}, L_{\infty}$ and so on.

Unfortunately, the above-mentioned strong properties of operators could be stated only for the intermediate spaces which are "distant" enough from the endpoint spaces of interpolation. The corresponding "measure" of this "distance" was proposed by D. Boyd in [1] by means of special indices, which must differ between intermediate and endpoint spaces; analogous conditions are crucial for all results from [10].

Weak type interpolation theorems with strict inequalities between the Boyd indices turn out to be useless in the so-called "extreme" (or "limiting") cases of various analytical problems, such as properties of integral transforms on Zygmund spaces $L \log L$ and $\exp L$, embeddings of Sobolev spaces $W_{p}^{k}(\Omega)$, when $p k=n$ (dimension of $\Omega$ ), etc. Generally speaking, we get here a problem of weak interpolation on intermediate spaces with the same Boyd indices as for the endpoint spaces. The pioneer work in this subject was done by Bennett and Rudnick in [3], where they introduced and studied the class of Lorentz-Zygmund spaces $L^{p, r}(\log L)^{\alpha}$. For example, one of the main results from [3] asserts that every quasilinear operator $T$ such that $T: L_{p_{0}, 1} \rightarrow L_{q_{0}, \infty}$ and $T: L_{p_{1}, 1} \rightarrow L_{q_{1}, \infty}$ with $0<p_{0}<p_{1} \leq \infty, 0<q_{0}<q_{1} \leq \infty$ acts from $L^{p_{1}, r}(\log L)^{\alpha}$ to $L^{q_{1}, r}(\log L)^{\alpha-1}$ for all $1 \leq r \leq \infty$ and all $\alpha \in \mathbb{R}$ such that $\frac{1}{r}<1-\alpha$.

The results of Bennett and Rudnick were later generalized to spaces with iterated $\log$ arithmic factors (like $L^{p_{1}, r}(\log L)^{\alpha}(\log \log L)^{\beta}$ in [7], [8] etc.) or to spaces $L_{p, \alpha, E}$ from [6], [12], where the exterior norm in $L_{r}$ was replaced by a norm in arbitrary space $\widetilde{E}$, rearrangement-invariant with respect to the homogeneous measure $\frac{d t}{t}$. All these spaces have additional parameterizations (the power of logarithm), giving a more delicate meter of proximity to the endpoint spaces. At the same time, the first parameter $p$, which corresponds to the power function $t^{1 / p}$ in the definition of norm in $L^{p, r}(\log L)^{\alpha}$ and $L_{p, \alpha, E}$, has no influence on interpolation and may be replaced by arbitrary increasing positive function $\phi(t)$. In result we obtain 
new spaces $L_{\phi, E}$ with the norm $\left\|\phi(t) f^{*}(t)\right\|_{\widetilde{E}}$ that were defined and studied in [13].

As known, any rearrangement-invariant space with the fundamental function $\phi(t)$ is intermediate between Lorentz space $\Lambda_{\phi}$ and Marcinkiewicz space $M_{\phi}$ with the same fundamental function (all definition will be given in the next section). The spaces $L_{\phi, E}$ coincide with those (and only those) rearrangement-invariant spaces that are interpolation between $\Lambda_{\phi}$ and $M_{\phi}$ and thus were named ultrasymmetric spaces. The behavior of these spaces in weak interpolation near endpoint spaces was investigated in [15] for operators of joint weak type $(a, b ; p, q)$ (in the sense of [4]) when the corresponding Boyd index of $L_{\phi, E}$ was equal to $1 / a$ or $1 / p$; many results from [15] can be easily generalized to operators of joint weak type $\left(\phi_{0}, \psi_{0} ; \phi_{1}, \psi_{1}\right)$ with functional parameters. Let us formulate one of such results.

Theorem 1.1. Let $\phi_{0}, \psi_{0}, \phi_{1}, \psi_{1}$ and also their ratios $\phi_{0} / \phi_{1}, \psi_{0} / \psi_{1}$ be bounded positive increasing functions on $(0,1)$ with positive extension indices. Let $\phi(t)=\mathbf{b}(t) \phi_{1}(t)$ where a bounded positive increasing function $\mathbf{b}(t)$ is such that $\mathbf{b}\left(t^{2}\right) \sim \mathbf{b}(t)$. Let $T$ be arbitrary (quasi)linear operator such that $T: \Lambda_{\phi_{0}} \rightarrow M_{\psi_{0}}, T: \Lambda_{\phi_{1}} \rightarrow M_{\psi_{1}}$. Then $T: L_{\phi, E} \rightarrow L_{\psi, E}$ with $\psi(t)=\mathbf{b}(t) \psi_{1}(t)\left(\ln \frac{e}{t}\right)^{-1}$ whenever the upper Boyd index $\rho_{E}<1$. The range space $L_{\psi, E}$ cannot be replaced here by any smaller ultrasymmetric space.

This assertion may be considered as optimal interpolation theorem but only among ultrasymmetric spaces. However we may expect better result, considering other types of rearrangement-invariant spaces. The indication to this can be seen in [12] where such better spaces were found for the partial case of spaces $L_{p, \alpha, E}$ which are ultrasymmetric with parameter function $\phi(t)=t^{1 / p}\left(\ln \frac{e}{t}\right)^{\alpha}$.

In the present paper we construct and study two new classes of rearrangement-invariant spaces different from ultrasymmetric spaces and providing improvement of Theorem 1.1 from two sides: they give larger domain and smaller range spaces. By analogy with [12] we call them $A$ and $B$ spaces; for some other purposes, analogous spaces were mentioned in [14]. Taking arbitrary quasi-power function $\phi(t), 0<t<1$ and arbitrary bounded positive increasing function $\mathbf{b}(t) \sim \mathbf{b}\left(t^{2}\right)$, we define the norm of the space $A_{\phi, \mathbf{b}, E}$ by the formula

$$
\|f\|_{A_{\phi, \mathbf{b}, E}}=\left\|\mathbf{b}(t) \int_{t}^{1} \phi(s) f^{* *}(s) \frac{d s}{s}\right\|_{\widetilde{E}}
$$


and the norm of the space $B_{\phi, \mathbf{b}, E}$ by the formula

$$
\|f\|_{B_{\phi, \mathbf{b}, E}}=\left\|\sup _{0<s<t} \phi(s) \mathbf{b}(s) f^{* *}(s)\right\|_{\widetilde{E}} .
$$

We study some general properties of these spaces: replacement $f^{* *}$ by $f^{*}$ in Section 3, interpolation properties in Section 4, fundamental functions in Section 5, compare new spaces with ultrasymmetric ones in Section 6 . Finally, Section 7 is devoted to weak type interpolation theorems improving Theorem 1.1. The basic definitions and notations as well as some properties of considered functions are collected in Section 2.

Throughout the paper we write $X=Y$ for spaces with equivalent (quasi)norms and $X \subset Y$ for continuous embedding, while $T: X \rightarrow Y$ will stand for the continuous operator acting from $X$ to $Y$. We shall write $f \lesssim g$ instead of $f \leq C g$ with some constant $C>0$ and $f \sim g$ if $f \lesssim g$ and $f \gtrsim g$ at the same time. Moreover, we do not distinguish the notions "increasing" and "non-decreasing" as well as "decreasing" and "non-increasing" and we say "a function $f$ is almost increasing (decreasing)" if it is equivalent to an increasing (decreasing) function.

\section{Preliminaries}

For the main definitions and properties concerning rearrangementinvariant spaces and interpolation theory, we refer the reader to the monographs [4] and [10]. Recall that a Banach function space $E=E(\Omega)$ of measurable functions $f: \Omega \mapsto \mathbb{R}^{n}$ is called rearrangement-invariant (r.i.) if $\|f\|_{E(\Omega)}=\left\|f^{*}\right\|_{E(0,|\Omega|)}$ for any $f \in E$ where, as usual,

$$
f^{*}(t)=\inf \{\lambda>0: \operatorname{mes}\{x \in \Omega:|f(x)|>\lambda\} \leq t\}, \quad t>0 .
$$

This definition allows us, without loss of generality, consider instead of an arbitrary set $\Omega$ only the one-dimensional interval $(0,|\Omega|)$. Moreover, we always may (and will) assume that any considered r.i. space $E$ is exact interpolation in the pair $\left(L_{1}, L_{\infty}\right)$. Hence $E=\left(L_{1}, L_{\infty}\right)_{D}^{K}$ for some suitable choice of parameter space $D$ (see [2]).

Let us recall more explicitly what this notation means. For any Banach pair $\left(A_{0}, A_{1}\right)$, one defines the Peetre $K$-functional for each $f \in A_{0}+A_{1}$ and each $t>0$ by

$$
K\left(t, f, A_{0}, A_{1}\right)=\inf _{f=f_{0}+f_{1}}\left\{\left\|f_{0}\right\|_{A_{0}}+t\left\|f_{1}\right\|_{A_{1}}\right\} .
$$

A Banach lattice $D$ of measurable functions on $(0, \infty)$ is called a parameter if it contains the function $\min (1, t)$. For each such $D$, the real interpolation 
space $\left(A_{0}, A_{1}\right)_{D}^{K}$ consists of all $f \in A_{0}+A_{1}$, for which

$$
\|f\|_{\left(A_{0}, A_{1}\right)_{D}^{K}}:=\left\|K\left(t, f, A_{0}, A_{1}\right)\right\|_{D}<\infty .
$$

In the present paper we will study interpolation properties of r.i. spaces $L_{\phi, E}, A_{\phi, \mathbf{b}, E}$ and $B_{\phi, \mathbf{b}, E}$ only for the case of bounded set $\Omega$ with standard Lebesgue measure, assuming, for simplicity, that $|\Omega|=1$. At the same time, for the parameter spaces $E$, we are going to work with two different measure spaces: $(0, \infty)$ with usual Lebesgue measure $d t$ and $(0,1)$ with homogeneous measure $\frac{d t}{t}$. If $E$ is a r.i. space on $((0, \infty), d t)$ then, following [6], we will denote by $\widetilde{E}$ the corresponding space on $\left((0,1), \frac{d t}{t}\right)$, constructed in the following way: $g \in \widetilde{E}$ if $f(t)=g\left(e^{-t}\right) \in E((0, \infty), d t)$. Setting $\|g\|_{\widetilde{E}}=\|f\|_{E}$, we obtain that $\widetilde{E}$ is an exact interpolation space in the Banach pair $\left(\widetilde{L}_{1}, \widetilde{L}_{\infty}\right)$, where

$$
\|g\|_{\widetilde{L}_{1}}=\int_{0}^{1}|g(t)| \frac{d t}{t}, \quad\|g\|_{\widetilde{L}_{\infty}}=\sup _{0<t<1}|g(t)|,
$$

which is constructed by the same interpolation functor as $E$ in the pair $\left(L_{1}, L_{\infty}\right)$. Some useful properties of space $\widetilde{E}$ can be found in [12].

Space $\widetilde{E}$ will be often supplied by some weight $w:(0,1) \rightarrow(0, \infty)$. We denote by $\widetilde{E}^{w}$ the space with the norm $\|f\|_{\widetilde{E}^{w}}=\|f w\|_{\widetilde{E}}$.

One of the basic characteristics of a r.i. space $E$ is its fundamental function $\varphi_{E}(\lambda)=\left\|\chi_{(0, \lambda)}\right\|_{E}$, which is continuous and quasiconcave. Moreover, the space $E$ always admits an equivalent renorming such that $\varphi_{E}(\lambda)$ becomes concave, so that $\varphi_{E}^{\prime}(\lambda)$ exists a.e. and is decreasing.

Another important characteristic of a r.i. space $E$ is its dilation function $d_{E}(t)$ which is defined as the norm of the dilation operator $\left(\sigma_{t} f\right)(s)=$ $f(s / t)$

$$
d_{E}(t)=\sup _{f \in E} \frac{\|f(s / t)\|_{E}}{\|f(s)\|_{E}}, \quad t>0
$$

The definition of $d_{E}(t)$ implies that

$$
\varphi_{E}(t s) \leq d_{E}(t) \varphi_{E}(s) \quad \text { for all } t, s>0
$$

(see [10, p. 102]). The dilation function is used for definition of the Boyd indices of the space $E$ :

$$
\pi_{E}=\lim _{t \rightarrow 0} \frac{\ln d_{E}(t)}{\ln t}, \quad \rho_{E}=\lim _{t \rightarrow \infty} \frac{\ln d_{E}(t)}{\ln t} .
$$

These indices always satisfy inequality $0 \leq \pi_{E} \leq \rho_{E} \leq 1$. 
In a similar way, given a positive increasing everywhere finite function $\varphi$ on $(0, \infty)$, we consider its extension function

$$
m_{\varphi}(t)=\sup _{s} \frac{\varphi(t s)}{\varphi(s)}
$$

and define the lower and the upper extension indices of $\varphi$ as

$$
\pi_{\varphi}=\lim _{t \rightarrow 0} \frac{\ln m_{\varphi}(t)}{\ln t}, \quad \rho_{\varphi}=\lim _{t \rightarrow \infty} \frac{\ln m_{\varphi}(t)}{\ln t} .
$$

In general $0 \leq \pi_{\varphi} \leq \rho_{\varphi}<\infty$, but $0 \leq \pi_{\varphi} \leq \rho_{\varphi} \leq 1$ if the function $\varphi$ is quasiconcave.

The Boyd indices of a r.i. space $E$ and the extension indices of its fundamental function satisfy inequalities $\pi_{E} \leq \pi_{\varphi_{E}}$ and $\rho_{E} \geq \rho_{\varphi_{E}}$. However, in many important cases (and among them all ultrasymmetric spaces) both kinds of indices coincide.

All the definitions above may be applied to r.i. spaces $G=G(0,1)$ if, for any function $f \in G$, to put $f(t)=0$ when $t>1$. We get that also $f^{*}(t)=0$ for $t>1$ and

$$
f^{* *}(t)= \begin{cases}\frac{1}{t} \int_{0}^{t} f^{*}(s) d s, & \text { for } t \leq 1, \\ \frac{1}{t} f^{* *}(1), & \text { for } t>1 .\end{cases}
$$

In result, $\varphi_{G}(t)=\varphi_{G}(1)$ for all $t>1$, and in the same vein we will extend any parameter function $\varphi(t)$. In order to have the extension indices $\pi_{\varphi}, \rho_{\varphi}$ depending only on values of $\varphi(t)$ for $t<1$, we define now the extension function as

$$
m_{\varphi}(t)=\sup _{0<s<1} \frac{\varphi(t s)}{\varphi(s)} .
$$

For any quasiconcave function $\varphi:(0,1) \rightarrow(0, \infty)$, we consider the Lorentz $\Lambda_{\varphi}$ and the Marcinkiewicz $M_{\varphi}$ spaces whose norms are

$$
\|f\|_{\Lambda_{\varphi}(0,1)}=\varphi(0+) f^{*}(0+)+\int_{0}^{1} f^{*}(u) d \varphi(u)
$$

and

$$
\|f\|_{M_{\varphi}(0,1)}=\sup _{0<u<1} \varphi(u) f^{* *}(u)
$$

respectively. (In fact, if $\varphi$ is not concave, $\|f\|_{\Lambda_{\varphi}(0,1)}$ is only a quasinorm equivalent to the norm $\|f\|_{\Lambda_{\tilde{\varphi}(0,1)}}$, where $\widetilde{\varphi}$ is a smallest concave majorant of $\varphi$. We will not differentiate such cases.) The characteristic function of both these spaces is $\varphi$ and the Boyd indices are equal to the upper and the lower extension indices of $\varphi$. 
When the underlying measure space is chosen to be $((0, \infty), d t)$, the corresponding Lorentz an Marcinkiewicz spaces $\Lambda_{\varphi}$ and $M_{\varphi}$ are defined analogously by the norms

$$
\begin{aligned}
\|f\|_{\Lambda_{\varphi}(0, \infty)} & =\varphi(0+) f^{*}(0+)+\int_{0}^{\infty} f^{*}(u) d \varphi(u), \\
\|f\|_{M_{\varphi}(0, \infty)} & =\sup _{0<u<\infty} \varphi(u) f^{* *}(u) .
\end{aligned}
$$

The spaces $\Lambda_{\varphi}$ and $M_{\varphi}$ are the smallest and the largest ones among all r.i. spaces with the same fundamental function, so that always $\Lambda_{\varphi_{G}} \subset G \subset M_{\varphi_{G}}$, i.e., any r.i. space $G$ is intermediate between $\Lambda_{\varphi_{G}}$ and $M_{\varphi_{G}}$. If, in addition, a space $G$ is interpolation between some $\Lambda_{\varphi}$ and $M_{\varphi}$, it will be named ultrasymmetric space. This class of spaces was introduced and studied in [13], where it was shown that a r.i. space $G$ is ultrasymmetric if and only if its norm is equivalent to $\left\|\varphi(t) f^{*}(t)\right\|_{\widetilde{E}}$ for some parameter space $\widetilde{E}$. This also implies that $\varphi_{G}(t) \sim \varphi(t)$. In result, the class of ultrasymmetric spaces contains such spaces as $L_{p}, L_{p, q}$, LorentzZygmund spaces from [3] and their generalizations from [7], [8] etc. When $\varphi(t)=t^{1 / p}\left(\ln \frac{e}{t}\right)^{\alpha}$, we get the spaces $L_{p, \alpha, E}$ studied in [12].

In order to show explicitly the parameters of an ultrasymmetric space $G$, we denote it by $L_{\varphi, E}$, setting

$$
\|f\|_{L_{\varphi, E}}:=\left\|\varphi(t) f^{*}(t)\right\|_{\widetilde{E}} .
$$

Note that, for the case of $\rho_{\varphi}<1$, it is shown in [6, Lemma 2.16] that $\left\|\varphi(t) f^{*}(t)\right\|_{\widetilde{E}} \sim\left\|\varphi(t) f^{* *}(t)\right\|_{\widetilde{E}}$. The Boyd indices of an ultrasymmetric space $G=L_{\varphi, E}$ are equal to extension indices of function $\varphi$, that is, $\pi_{G}=\pi_{\varphi}, \rho_{G}=\rho_{\varphi}$. Other properties of these spaces can be found in [13].

In this paper we shall study the spaces $L_{\varphi, E}$ for the case when $\varphi:(0,1) \rightarrow$ $(0, \infty)$ is a quasi-power function, that is $\varphi$ is quasiconcave and

$$
\varphi(t) \sim \int_{0}^{1} \min \left(1, \frac{t}{s}\right) \varphi(s) \frac{d s}{s}, \quad \text { for all } t \in(0, \infty) .
$$

It can be proved that $\varphi$ is a quasi-power function if and only if $0<\pi_{\varphi} \leq$ $\rho_{\varphi}<1$ (see [10, p. 57]). We consider interpolation (and some other) properties of families of spaces $L_{\varphi, E}$ with $\varphi(t)=\phi(t) \mathbf{b}(t)$, where the first factor $\phi(t)$ is the same for all spaces while $\mathbf{b}(t):(0,1) \rightarrow(0, \infty)$ is arbitrary bounded positive increasing function such that $\mathbf{b}\left(t^{2}\right) \sim \mathbf{b}(t)$. This condition implies immediately that

$$
m_{\mathbf{b}}(t)=\sup _{s} \frac{\mathbf{b}(t s)}{\mathbf{b}(s)} \sim \sup _{s} \frac{\mathbf{b}\left(t^{2} s^{2}\right)}{\mathbf{b}\left(s^{2}\right)}=m_{\mathbf{b}}\left(t^{2}\right),
$$


and submultiplicativity of $m_{\mathbf{b}}(t)$ implies that $m_{\mathbf{b}}(t) \sim 1$. Consequently, $\pi_{\mathbf{b}}=\rho_{\mathbf{b}}=0$. In particular, this gives that the function $\frac{\mathbf{b}(t)}{t^{\alpha}}$ is almost decreasing for any $\alpha>0$.

For every function $\mathbf{b}$ as above, we define a new function $\mathbf{B}$ by the formula $\mathbf{b}(t)=\mathbf{B}\left(\frac{1}{\ln \frac{e}{t}}\right)$, or equivalently by $\mathbf{B}(u)=\mathbf{b}\left(e^{1-\frac{1}{u}}\right)$. In result, $\mathbf{B}(u)$ is also positive and increasing. The condition $\mathbf{b}\left(t^{2}\right) \sim \mathbf{b}(t)$ on function $\mathbf{b}$ implies that the function $\mathbf{B}$ satisfies the $\Delta_{2}$ condition, $\mathbf{B}(t) \sim \mathbf{B}(2 t)$, so that $m_{\mathbf{B}}(t)<\infty$ for all $t>0$. Hence there exist the extension indices $\pi_{\mathbf{B}}, \rho_{\mathbf{B}}$ and $0 \leq \pi_{\mathbf{B}} \leq \rho_{\mathbf{B}}<\infty$. The case of $\pi_{\mathbf{B}}=0$ requires special consideration because the function $\mathbf{b}(t)$ becomes too slow (like $\ln |\ln (t)|$ ); that is why we always assume that $\pi_{\mathbf{B}}>0$.

In order to stress the role of either of factors $\phi, \mathbf{b}$ we will write $L_{\phi, \mathbf{b}, E}$ instead of $L_{\phi \mathbf{b}, E}$; analogously $M_{\phi, \mathbf{b}}$ will stand for the Marcinkiewicz space $M_{\phi \mathbf{b}}$ and so on. It is easy to see that $\pi_{\phi \mathbf{b}}=\pi_{\phi}$ and $\rho_{\phi \mathbf{b}}=\rho_{\phi}$ and hence the Boyd indices of the space $L_{\phi, \mathbf{b}, E}$ are equal to the extension indices of the function $\phi$. Moreover, the function $\phi \mathbf{b}$ is also a quasi-power function and then $\frac{\phi(t) \mathbf{b}(t)}{t}$ is an almost decreasing function (see [10, p. 55]).

Recall that we do not differentiate spaces with equivalent norms. This allows us to replace any of parameter functions $\phi, \psi, \mathbf{b}, \mathbf{B}$ etc. by equivalent one without change of the corresponding space. For example, we may always regard the functions $\phi(t) / t, \mathbf{b}(t) / t$ etc. as strictly decreasing, omitting the word "almost".

The following lemmas will be useful in the sequel.

Lemma 2.1. Let $E$ be an r.i. space with $\rho_{E}<\pi_{\mathbf{B}}$, then

$$
\left\|\mathbf{b}\left(t e^{-u}\right)\right\|_{E} \sim \varphi_{E}\left(\ln \frac{e}{t}\right) \mathbf{b}(t), \quad \text { for all } t \in(0,1), u \in(0, \infty) .
$$

Proof. The estimate in one direction is always true. Indeed, since $\mathbf{b}$ is increasing, $\mathbf{b}(t) / t$ is decreasing and $\mathbf{b}\left(t^{2}\right) \sim \mathbf{b}(t)$, one obtains

$$
\begin{aligned}
\left\|\mathbf{b}\left(t e^{-u}\right)\right\|_{E} & \geq\left\|\mathbf{b}\left(t e^{-u}\right) \chi_{\left(0, \ln \frac{e}{t}\right)}(u)\right\|_{E} \\
& \geq\left\|\mathbf{b}\left(\frac{t^{2}}{e}\right) \chi_{\left(0, \ln \frac{e}{t}\right)}(u)\right\|_{E} \\
& \gtrsim \mathbf{b}(t) \varphi_{E}\left(\ln \frac{e}{t}\right)
\end{aligned}
$$

For the estimate in the other direction, let us suppose that the function $\varphi_{E}$ is concave. Hence $\Lambda_{\varphi_{E}} \subset E$ and

$$
\left\|b\left(t e^{-u}\right)\right\|_{E} \leq\left\|b\left(t e^{-u}\right)\right\|_{\Lambda \phi_{E}}=\varphi_{E}(0+) \mathbf{b}(t)+\int_{0}^{\infty} \mathbf{b}\left(t e^{-u}\right) \varphi_{E}^{\prime}(u) d u .
$$


But $\varphi_{E}(0+) \leq \varphi_{E}\left(\ln \frac{e}{t}\right)$ for any $t<1$, hence we have to estimate only the last integral. It may be presented as sum of two separate integrals over the intervals $\left(0, \ln \frac{e}{t}\right)$ and $\left(\ln \frac{e}{t}, \infty\right)$. For the first one we have

$$
\int_{0}^{\ln \frac{e}{t}} \mathbf{b}\left(t e^{-u}\right) \varphi_{E}^{\prime}(u) d u \leq \mathbf{b}(t) \int_{0}^{\ln \frac{e}{t}} \varphi_{E}^{\prime}(u) d u \leq \mathbf{b}(t) \varphi_{E}\left(\ln \frac{e}{t}\right) .
$$

In order to estimate the integral over $\left(\ln \frac{e}{t}, \infty\right)$ we use the inequality $\varphi_{E}^{\prime}(s) \leq \varphi_{E}(s) / s$, which is valid because of the concavity of $\varphi_{E}(s)$. Then, changing variables, we get

$$
\begin{aligned}
\int_{\ln \frac{e}{t}}^{\infty} \mathbf{b}\left(t e^{-u}\right) \varphi_{E}^{\prime}(u) d u & \leq \int_{\ln \frac{e}{t}}^{\infty} \mathbf{b}\left(t e^{-u}\right) \varphi_{E}(u) \frac{d u}{u} \\
& =\int_{1}^{\infty} \mathbf{b}\left(t e^{-v \ln \frac{e}{t}}\right) \varphi_{E}\left(v \ln \frac{e}{t}\right) \frac{d v}{v} .
\end{aligned}
$$

Now, by definition of $\mathbf{B}$ and taking into account that $\mathbf{b}$ is increasing, we have for all $v \in(1, \infty)$

$$
\mathbf{b}\left(t e^{-v \ln \frac{e}{t}}\right) \leq \mathbf{b}\left(t e^{-(v-1) \ln \frac{e}{t}}\right)=\mathbf{B}\left(\frac{1}{v \ln \frac{e}{t}}\right) \leq m_{\mathbf{B}}\left(\frac{1}{v}\right) \mathbf{b}(t) .
$$

Thus, using also (2.1), we obtain

$$
\int_{1}^{\infty} \mathbf{b}\left(t e^{-v \ln \frac{e}{t}}\right) \varphi_{E}\left(v \ln \frac{e}{t}\right) \frac{d v}{v} \leq \varphi_{E}\left(\ln \frac{e}{t}\right) \mathbf{b}(t) \int_{1}^{\infty} m_{\mathbf{B}}\left(\frac{1}{v}\right) d_{E}(v) \frac{d v}{v} .
$$

The last integral converges because $\rho_{E}<\pi_{\mathbf{B}}$ and the proof is finished.

Lemma 2.2. If $\pi_{\mathrm{B}}>0$, then

$$
\int_{0}^{u} \frac{1}{\mathbf{b}\left(e^{-v}\right)(1+v)} d v \lesssim \frac{1}{\mathbf{b}\left(e^{-u}\right)}, \quad \text { for all } u \in(0, \infty) .
$$

Proof. Changing variables, $\frac{1}{1+v}=t$, we obtain

$$
\int_{0}^{u} \frac{1}{\mathbf{b}\left(e^{-v}\right)(1+v)} d v=\int_{0}^{u} \frac{1}{\mathbf{B}\left(\frac{1}{1+v}\right)(1+v)} d v=\int_{\frac{1}{1+u}}^{1} \frac{1}{\mathbf{B}(t)} \frac{d t}{t} .
$$

Let us take $\alpha$ such that $0<\alpha<\pi_{\mathbf{B}}$. Then $B(t) t^{-\alpha}$ is almost increasing and hence

$$
\int_{\frac{1}{1+u}}^{1} \frac{1}{\mathbf{B}(t)} \frac{d t}{t} \lesssim \frac{1}{\mathbf{B}\left(\frac{1}{1+u}\right)}\left(\frac{1}{1+u}\right)^{\alpha} \int_{\frac{1}{1+u}}^{1} \frac{d t}{t^{1+\alpha}} \lesssim \frac{1}{\mathbf{B}\left(\frac{1}{1+u}\right)}=\frac{1}{\mathbf{b}\left(e^{-u}\right)}
$$


Lemma 2.3. If $\pi_{\mathbf{B}}>1$, then

$$
\int_{u}^{\infty} \mathbf{b}\left(e^{-v}\right) d v \sim \mathbf{b}\left(e^{-u}\right)(1+u), \quad \text { for all } u \in(0, \infty) .
$$

Proof. Changing variables as in Lemma 2.2, we get

$$
\int_{u}^{\infty} \mathbf{b}\left(e^{-v}\right) d v=\int_{0}^{\frac{1}{1+u}} \frac{\mathbf{B}(t)}{t} \frac{d t}{t} .
$$

Let us consider now $\psi(t)=\frac{\mathbf{B}(t)}{t}$. Then we have $0<\pi_{\psi}=\pi_{\mathbf{B}}-1 \leq$ $\rho_{\mathbf{B}}-1=\rho_{\psi}<\infty$ and (see [10, p. 57])

$$
\int_{0}^{s} \psi(t) \frac{d t}{t} \sim \psi(s)
$$

that immediately leads to the desired equivalence.

Remark 2.4. Sometimes it is more convenient to rewrite the relations (2.3) and (2.4) in the form

$$
\int_{t}^{1} \frac{1}{\mathbf{b}(s) \ln \frac{e}{s}} \frac{d s}{s} \lesssim \frac{1}{\mathbf{b}(t)} \quad \text { and } \quad \int_{0}^{t} \mathbf{b}(s) \frac{d s}{s} \sim \mathbf{b}(t) \ln \frac{e}{t}, \quad \forall t \in(0,1),
$$

respectively.

\section{Definition of spaces $A$ and $B$}

For $1<p \leq \infty$ and $\alpha \in \mathbb{R}$, the $A$ and $B$ type spaces $A_{p, \alpha, E}, B_{p, \alpha, E}$ are defined in [12]. The main advantage of these spaces is that they appear in a natural way as optimal domain and range spaces, respectively, in the corresponding weak type interpolation theorems. Moreover, they are rather different from classical rearrangement invariant spaces. In these section we generalize and study these spaces for any quasi-power function $\phi$ and any bounded increasing function $\mathbf{b}$, such that $\mathbf{b}\left(t^{2}\right) \sim \mathbf{b}(t)$.

The space $A_{\phi, \mathbf{b}, E}$ is defined as a collection of all measurable functions $f$ on $(0,1)$, having the finite norm

$$
\|f\|_{A_{\phi, \mathbf{b}, E}}=\left\|\mathbf{b}(t) \int_{t}^{1} \phi(s) f^{* *}(s) \frac{d s}{s}\right\|_{\widetilde{E}} .
$$

Observe that this definition makes sense not for every r.i. space $E$. Indeed, as any r.i. space on the interval $(0,1)$ the space $A_{\phi, \mathbf{b}, E}$ must contain all bounded functions, in particular, the function $\chi_{(0,1)}$. 
Lemma 3.1. The space $A_{\phi, \mathbf{b}, E}$ contains the function $\chi_{(0,1)}$ (and thus is non-trivial) if and only if $\mathbf{b}\left(e^{-u}\right) \in E$.

Proof. Using the fact that $\phi$ is a quasi-power function, we obtain that

$$
\left\|\chi_{(0,1)}\right\|_{A_{\phi, \mathbf{b}, E}}=\left\|\mathbf{b}(t) \int_{t}^{1} \phi(s) \frac{d s}{s}\right\|_{\widetilde{E}} \lesssim \phi(1)\|b(t)\|_{\widetilde{E}}, \quad \text { for all } t \in(0,1) .
$$

Hence, if $\mathbf{b}(t) \in \widetilde{E}$, that is $\mathbf{b}\left(e^{-u}\right) \in E$, then $\chi_{(0,1)} \in A_{\phi, \mathbf{b}, E}$.

Conversely,

$$
\left\|\chi_{(0,1)}\right\|_{A_{\phi, \mathbf{b}, E}} \geq\left\|\chi_{\left(0, \frac{1}{2}\right)}(t) \mathbf{b}(t) \int_{\frac{1}{2}}^{1} \phi(s) \frac{d s}{s}\right\|_{\widetilde{E}} \gtrsim \phi(1)\left\|\chi_{\left(0, \frac{1}{2}\right)}(t) \mathbf{b}(t)\right\|_{\widetilde{E}}
$$

due to the function $\phi(t) / t$ being decreasing. Thus we obtain that $\chi_{\left(0, \frac{1}{2}\right)}(t) \mathbf{b}(t) \in \widetilde{E}$. On the other hand, $\chi_{\left(\frac{1}{2}, 1\right)}(t) \mathbf{b}(t) \in \widetilde{E}$ owing to Lemma 1.1 from [12]. Hence the sum function $\mathbf{b}(t)$ belongs to $\widetilde{E}$, i.e. $\mathbf{b}\left(e^{-u}\right) \in E$.

As a partial case, the A-type spaces $A_{\phi, \mathbf{b}, E}$ include the spaces $A_{p, \alpha, E}$ from [12] when $\phi(t)=t^{\frac{1}{p}}$ and $\mathbf{b}(t)=\left(\ln \frac{e}{t}\right)^{\alpha-1}, \alpha<1$. If, in addition, $E=L_{r}$ then they coincide with the spaces $\mathcal{L}^{p, r}(\log \mathcal{L})^{\alpha-1}$, defined by Bennett and Rudnick in [3].

For some of future applications, it will be useful to state the following equivalence between the norm (3.1) and the quasi-norm obtained via replacing $f^{* *}$ by $f^{*}$.

Lemma 3.2. For any r.i. space $E$, one has

$$
\|f\|_{A_{\phi, \mathbf{b}, E}} \sim\left\|\mathbf{b}(t) \int_{t}^{1} \phi(s) f^{*}(s) \frac{d s}{s}\right\|_{\widetilde{E}} .
$$

Proof. Since always $f^{*}(t) \leq f^{* *}(t)$, it is enough to prove the inequality with $\lesssim$. In order to do this we consider first the integral

$$
\begin{aligned}
\int_{t}^{1} \phi(s) f^{* *}(s) \frac{d s}{s} & =\int_{t}^{1} \phi(s)\left(\int_{0}^{s} f^{*}(u) d u\right) \frac{d s}{s^{2}} \\
& =\int_{0}^{t} f^{*}(u) d u\left(\int_{t}^{1} \phi(s) \frac{d s}{s^{2}}\right)+\int_{t}^{1} f^{*}(u)\left(\int_{u}^{1} \phi(s) \frac{d s}{s^{2}}\right) d u \\
& \lesssim \frac{\phi(t)}{t} \int_{0}^{t} f^{*}(u) d u+\int_{t}^{1} f^{*}(u) \frac{\phi(u)}{u} d u \\
& =\phi(t) f^{* *}(t)+\int_{t}^{1} f^{*}(u) \phi(u) \frac{d u}{u}
\end{aligned}
$$


where we again use the quasi-power character of the function $\phi$. Hence

$$
\begin{aligned}
\left\|\mathbf{b}(t) \int_{t}^{1} \phi(s) f^{* *}(s) \frac{d s}{s}\right\|_{\widetilde{E}} \lesssim & \left\|\mathbf{b}(t) \phi(t) f^{* *}(t)\right\|_{\widetilde{E}} \\
& +\left\|\mathbf{b}(t) \int_{t}^{1} \phi(u) f^{*}(u) \frac{d u}{u}\right\|_{\widetilde{E}}
\end{aligned}
$$

On the other hand, applying Lemma 1.2 from [12] with $a=\frac{1}{2}, b=1$, we have that

$$
\left\|\mathbf{b}(t) \int_{t}^{1} \phi(u) f^{*}(u) \frac{d u}{u}\right\|_{\widetilde{E}} \gtrsim\left\|\mathbf{b}\left(\frac{t}{2}\right) \int_{t / 2}^{1} \phi(u) f^{*}(u) \frac{d u}{u}\right\|_{\widetilde{E}} .
$$

Using that $\mathbf{b}(t) / t$ and $f^{*}$ are decreasing, that $\int_{t / 2}^{t} \frac{\phi(s)}{s} d s \geq \frac{\phi(t)}{t}\left(t-\frac{t}{2}\right)=$ $\frac{\phi(t)}{2}$ and Lemma 2.16 from [6], we obtain that

$$
\begin{aligned}
\left\|\mathbf{b}(t) \int_{t}^{1} \phi(u) f^{*}(u) \frac{d u}{u}\right\|_{\widetilde{E}} & \gtrsim\left\|\mathbf{b}(t) \int_{t / 2}^{t} \phi(u) f^{*}(u) \frac{d u}{u}\right\|_{\widetilde{E}} \\
& \gtrsim\left\|\mathbf{b}(t) \phi(t) f^{*}(t)\right\|_{\widetilde{E}} \\
& \sim\left\|\mathbf{b}(t) \phi(t) f^{* *}(t)\right\|_{\widetilde{E}} .
\end{aligned}
$$

Thus the first summand in the right-hand side of $(3.2)$ is almost less than the second one that gives the desired estimate with $\lesssim$.

Remark 3.3. The last inequality implies that $A_{\phi, \mathbf{b}, E} \subseteq L_{\phi, \mathbf{b}, E}$.

The space $B_{\phi, \mathbf{b}, E}$ is defined as a collection of all measurable functions $f(t)$ on $(0,1)$ for which the norm

$$
\|f\|_{B_{\phi, \mathbf{b}, E}}=\left\|\sup _{0<s<t} \phi(s) \mathbf{b}(s) f^{* *}(s)\right\|_{\widetilde{E}}
$$

is finite.

When $\phi(t)=t^{\frac{1}{q}}$ and $\mathbf{b}(t)=\left(\ln \frac{e}{t}\right)^{\alpha-1}, \alpha<1$, these spaces coincide with $B$ spaces $B_{q, \alpha, E}$ defined in [12] (and in [6] when $\alpha=0$ ).

Like $A$-type spaces, the norm in $B_{\phi, \mathbf{b}, E}$ is equivalent to a quasi-norm obtained via replacing $f^{* *}$ by $f^{*}$.

Lemma 3.4. For any r.i. space $E$ we have

$$
\|f\|_{B_{\phi, \mathbf{b}, E}} \sim\left\|\sup _{0<s<t} \phi(s) \mathbf{b}(s) f^{*}(s)\right\|_{\widetilde{E}}
$$

Proof. Again it is enough to prove the upper estimate

$$
\|f\|_{B_{\phi, \mathbf{b}, E}} \lesssim\left\|\sup _{0<s<t} \phi(s) \mathbf{b}(s) f^{*}(s)\right\|_{\widetilde{E}} .
$$


Defining the function $h(t)=\sup _{0<s<t} \phi(s) \mathbf{b}(s) f^{*}(s)$, we get for all $s<t$ the estimate

$$
f^{*}(s) \leq \frac{1}{\phi(s) \mathbf{b}(s)} h(t)
$$

hence

$$
f^{* *}(s)=\frac{1}{s} \int_{0}^{s} f^{*}(u) d u \leq \frac{h(t)}{s} \int_{0}^{s} \frac{d u}{\phi(u) \mathbf{b}(u)} .
$$

Substituting into the norm expression, we obtain that

$$
\begin{aligned}
\|f\|_{B_{\phi, \mathbf{b}, E}} & \leq\left\|\sup _{0<s<t} \frac{\phi(s) \mathbf{b}(s)}{s} h(t) \int_{0}^{s} \frac{d u}{\phi(u) \mathbf{b}(u)}\right\|_{\widetilde{E}} \\
& \leq\left(\sup _{0<s<1} \frac{\phi(s) \mathbf{b}(s)}{s} \int_{0}^{s} \frac{d u}{\phi(u) \mathbf{b}(u)}\right)\|h\|_{\widetilde{E}} .
\end{aligned}
$$

Thus we only have to prove that the expression in the round brackets is finite. In order to do this we define the function $\psi(s)=\frac{s}{\phi(s) \mathbf{b}(s)}$ which is a quasi-power, since $0<1-\rho_{\phi \mathbf{b}}=\pi_{\psi} \leq \rho_{\psi}=1-\pi_{\phi \mathbf{b}}<1$. Hence (see [10, p. 57])

$$
\int_{0}^{s} \frac{d u}{\phi(u) \mathbf{b}(u)} \sim \frac{s}{\phi(s) \mathbf{b}(s)}
$$

and (3.3) is proved.

The main distinction of the norm in the space $B_{\phi, \mathbf{b}, E}$ from that in the corresponding ultrasymmetric space $L_{\phi, \mathbf{b}, E}$ consists in taking the least increasing majorant before computing the exterior norm, hence always $B_{\phi, \mathbf{b}, E} \subset L_{\phi, \mathbf{b}, E}$. As we will see after computing the fundamental functions of both types of spaces in Section 5 below, this embedding is proper whenever $\lim _{t \rightarrow \infty} \varphi_{E}(t)=\infty$, i.e., $E \nsubseteq L_{\infty}$.

Proposition 3.5. Suppose that the r.i. space E satisfies the condition

$$
\lim _{t \rightarrow \infty} \varphi_{E}(t)<\infty .
$$

Then $B_{\phi, \mathbf{b}, E}=L_{\phi, \mathbf{b}, E}$

Proof. It is enough to show that $L_{\phi, \mathbf{b}, E} \subseteq B_{\phi, \mathbf{b}, E}$ when $E$ satisfies (3.4). Given $f \in L_{\phi, \mathbf{b}, E}$, we introduce the functions

$$
F(u)=\phi\left(e^{-u}\right) \mathbf{b}\left(e^{-u}\right) f^{* *}\left(e^{-u}\right) \quad \text { and } \quad H(u)=\sup _{u<v} F(v) .
$$

We see that $F \in E$, since $\|f\|_{L_{\phi, \mathbf{b}, E}} \sim\|F\|_{E}$. Moreover, $\|H\|_{E}=$ $\|f\|_{B_{\phi, \mathbf{b}, E}}$, hence it remains to show that $\|H\|_{E} \lesssim\|F\|_{E}$. 
Using the properties of $\phi$ and $\mathbf{b}$, we can prove that, for each fixed $u>0$ and all $v \in(u, u+1)$, we have

$$
F(v) \gtrsim \phi\left(e^{-u}\right) \mathbf{b}\left(e^{-(u+1)}\right) f^{* *}\left(e^{-u}\right) \gtrsim F(u) .
$$

Then, for each $u>0$,

$$
\|f\|_{L_{\phi, \mathbf{b}, E}} \gtrsim\left\|F \chi_{(u, u+1)}\right\|_{E} \gtrsim F(u)\left\|\chi_{(0,1)}\right\|_{E},
$$

so that $F \in L_{\infty}$ and the same happens with $H$. Moreover, we have the norm estimate

$$
\|H\|_{L_{\infty}}=\|F\|_{L_{\infty}} \lesssim \frac{1}{\left\|\chi_{(0,1)}\right\|_{E}}\|f\|_{L_{\phi, \mathbf{b}, E}}
$$

Now, applying the same arguments as in the proof of Theorem 6.8 from [6], we obtain that $\|H\|_{E} \lesssim\|F\|_{E}$ as required.

\section{Interpolation property of $A$ and $B$ spaces}

In the first part of this section we study interrelation between the spaces $A_{\phi, \mathbf{b}, E}$ and some real interpolation spaces in the Banach pair $\left(\Lambda_{\phi}, A_{\phi, \mathbf{b}}\right)$, where $A_{\phi, \mathbf{b}}$ stands for the extreme space $A_{\phi, \mathbf{b}, L_{\infty}}$ with the norm

$$
\|f\|_{A_{\phi, \mathbf{b}}}=\sup _{0<t<1} \mathbf{b}(t) \int_{t}^{1} \phi(s) f^{* *}(s) \frac{d s}{s} .
$$

Let us start with studying the $K$-functional in this pair.

Lemma 4.1. For all $x \in(0,1)$ and any $f \in \Lambda_{\phi}+A_{\phi, \mathbf{b}}$,

$$
K\left(\frac{1}{\mathbf{b}(x)}, f, \Lambda_{\phi}, A_{\phi, \mathbf{b}}\right) \lesssim \frac{1}{\mathbf{b}(x)} \cdot \sup _{0<t<x} \mathbf{b}(t) \int_{t}^{1} \phi(s) f^{* *}(s) \frac{d s}{s} .
$$

Proof. Let $f$ be a function in $\Lambda_{\phi}+A_{\phi, \mathbf{b}}$ and $x \in(0,1)$. We decompose it as $f(s)=f_{0}(s)+f_{1}(s)$ where

$$
\begin{aligned}
& f_{0}(s)=\min \left\{|f(s)|, f^{*}(x)\right\} \operatorname{sign} f(s), \\
& f_{1}(s)=\left[\max \left\{|f(s)|, f^{*}(x)\right\}-f^{*}(x)\right] \operatorname{sign} f(s) .
\end{aligned}
$$

From the definition of $K$-functional we have

$$
K\left(\frac{1}{\mathbf{b}(x)}, f, \Lambda_{\phi}, A_{\phi, \mathbf{b}}\right) \leq\left\|f_{0}\right\|_{\Lambda_{\phi}}+\frac{1}{\mathbf{b}(x)}\left\|f_{1}\right\|_{A_{\phi, \mathbf{b}}} .
$$


Then in order to prove the lemma it is sufficient to compare separately $\left\|f_{0}\right\|_{\Lambda_{\phi}}$ and $\frac{1}{\mathbf{b}(x)}\left\|f_{1}\right\|_{A_{\phi, \mathbf{b}}}$ with the right-hand side of (4.2), namely, with $\sup _{0<t<x} R(t)$, where

$$
R(t)=\frac{\mathbf{b}(t)}{\mathbf{b}(x)} \int_{t}^{1} \phi(s) f^{* *}(s) \frac{d s}{s} .
$$

First we consider the norm $\left\|f_{0}\right\|_{\Lambda_{\phi}}$ and separate it into two integrals

$$
\left\|f_{0}\right\|_{\Lambda_{\phi}}=\int_{0}^{x} \phi(s) f^{*}(x) \frac{d s}{s}+\int_{x}^{1} \phi(s) f^{*}(s) \frac{d s}{s}=I_{1}+I_{2} .
$$

The integral $I_{2}$ is estimated as follows:

$$
I_{2}=\int_{x}^{1} \phi(s) f^{*}(s) \frac{d s}{s} \leq \int_{x}^{1} \phi(s) f^{* *}(s) \frac{d s}{s}=R(x) \leq \sup _{0<t<x} R(t) .
$$

In order to estimate $I_{1}$ we assume first that $x \leq 1 / 2$. Since the functions $s f^{* *}(s)$ and $\phi$ are increasing, we obtain that

$$
\begin{aligned}
\sup _{0<t<x} R(t) \geq R(x) & =\int_{x}^{1} \phi(s)\left(s f^{* *}(s)\right) \frac{d s}{s^{2}} \\
& \geq x f^{* *}(x) \phi(x) \int_{x}^{1} \frac{d s}{s^{2}} \\
& =x f^{* *}(x) \phi(x)\left(\frac{1}{x}-1\right) \\
& =f^{* *}(x) \phi(x)(1-x) \\
& \gtrsim f^{* *}(x) \phi(x)
\end{aligned}
$$

Moreover, since $\phi$ is a quasi-power function, we have

$$
I_{1}=f^{*}(x) \int_{0}^{x} \phi(s) \frac{d s}{s} \lesssim f^{* *}(x) \phi(x) .
$$

Hence, combining both inequalities, we obtain that $I_{1} \lesssim \sup _{0<t<x} R(t)$ when $x \leq 1 / 2$.

If $1 / 2<x<1$ then

$$
\begin{aligned}
\sup _{0<t<x} R(t) & \geq R\left(\frac{1}{2}\right)=\frac{\mathbf{b}\left(\frac{1}{2}\right)}{\mathbf{b}(x)} \int_{\frac{1}{2}}^{1} \phi(s)\left(s f^{* *}(s)\right) \frac{d s}{s^{2}} \\
& \gtrsim f^{* *}\left(\frac{1}{2}\right) \phi\left(\frac{1}{2}\right) \int_{\frac{1}{2}}^{1} \frac{d s}{s^{2}} \gtrsim f^{* *}(x) \phi(x),
\end{aligned}
$$


since the functions $t f^{* *}(t)$ and $\phi$ are increasing and the functions $\phi(t) / t$, $\mathbf{b}(t) / t$ and $f^{* *}$ are decreasing. Thus we obtain that, in the case of $1 / 2<x<1$, the inequality

$$
\sup _{0<t<x} R(t) \gtrsim f^{* *}(x) \phi(x) \geq I_{1}
$$

holds as well. Summing up, we obtain that, whenever $x \in(0,1)$,

$$
\left\|f_{0}\right\|_{\Lambda_{\phi}} \leq I_{1}+I_{2} \lesssim \sup _{0<t<x} R(t) .
$$

Now we pass to estimating the norm $\left\|f_{1}\right\|_{A_{\phi, \mathbf{b}}}$ :

$$
\left\|f_{1}\right\|_{A_{\phi, \mathbf{b}}}=\sup _{0<t<1} \mathbf{b}(t) \int_{t}^{1} \phi(s) f_{1}^{* *}(s) \frac{d s}{s}=\max \left(\xi_{1}, \xi_{2}\right),
$$

where

$$
\xi_{1}=\sup _{0<t<x} \mathbf{b}(t) \int_{t}^{1} \phi(s) f_{1}^{* *}(s) \frac{d s}{s}, \quad \xi_{2}=\sup _{x<t<1} \mathbf{b}(t) \int_{t}^{1} \phi(s) f_{1}^{* *}(s) \frac{d s}{s} .
$$

Since $f_{1}^{*}(s) \leq f^{*}(s)$ for all $s \in(0,1)$, we obtain that

$$
\xi_{1} \leq \sup _{0<t<x} \mathbf{b}(t) \int_{t}^{1} \phi(s) f^{* *}(s) \frac{d s}{s}=\mathbf{b}(x) \cdot \sup _{0<t<x} R(t),
$$

so we only need to estimate the second supremum. For all $t \in[x, 1)$, the function $f_{1}^{*}(t)=0$, thus

$$
\begin{aligned}
\xi_{2} & =\sup _{x<t<1} \mathbf{b}(t) \int_{t}^{1} \phi(s)\left(\int_{0}^{x} f_{1}^{*}(u) d u\right) \frac{d s}{s^{2}} \\
& \leq\left(\int_{0}^{x} f^{*}(u) d u\right) \sup _{x<t<1} \mathbf{b}(t) \int_{t}^{1} \phi(s) \frac{d s}{s^{2}} \\
& \lesssim x f^{* *}(x) \sup _{x<t<1} \mathbf{b}(t) \frac{\phi(t)}{t}
\end{aligned}
$$

where we use again the fact that $\phi$ is a quasi-power function. Moreover, the function $\frac{\phi(t) \mathbf{b}(t)}{t}$ is almost decreasing and we know that $f^{* *}(x) \phi(x) \lesssim$ $\sup _{0<t<x} R(t)$ by (4.4) for $x \leq 1 / 2$ and by (4.5) for $x>1 / 2$, hence

$$
\xi_{2} \lesssim f^{* *}(x) \mathbf{b}(x) \phi(x) \lesssim \mathbf{b}(x) \cdot \sup _{0<t<x} R(t)
$$


Consequently, all components of the right-hand side of equation (4.3) are almost less than $\sup _{0<t<x} R(t)$ and the proof is complete.

As a consequence of the estimate (4.2), we obtain the desired interpolation property of the $A$-type spaces.

Theorem 4.2. Let $D$ be a r.i. space defined by $\|f\|_{D}=\|F\|_{E}$, where $F(u)=f\left(\frac{1}{\mathbf{b}\left(e^{-u}\right)}\right), 0<u<\infty$, then

$$
A_{\phi, \mathbf{b}, E} \subseteq\left(\Lambda_{\phi}, A_{\phi, \mathbf{b}}\right)_{D^{w}}^{K}, \quad \text { with } w(u)=\frac{1}{u} .
$$

Proof. We have that

$$
\begin{aligned}
\|f\|_{\left(\Lambda_{\phi}, A_{\phi, \mathbf{b}}\right)_{D}^{K} w} & =\left\|\frac{1}{z} K\left(z, f, \Lambda_{\phi}, A_{\phi, \mathbf{b}}\right)\right\|_{D} \\
& =\left\|\mathbf{b}\left(e^{-z}\right) K\left(\frac{1}{\mathbf{b}\left(e^{-z}\right)}, f, \Lambda_{\phi}, A_{\phi, \mathbf{b}}\right)\right\|_{E} \\
& \lesssim\left\|\sup _{0<t<e^{-z}} \mathbf{b}(t) \int_{t}^{1} \phi(s) f^{* *}(s) \frac{d s}{s}\right\|_{E} \\
& =\left\|\sup _{u>z} \mathbf{b}\left(e^{-u}\right) \int_{e^{-u}}^{1} \phi(s) f^{* *}(s) \frac{d s}{s}\right\|_{E} .
\end{aligned}
$$

Now we may use Theorem 4.2 from [12], taking there

$$
g(u)=\mathbf{b}\left(e^{-u}\right), \quad h(u)=\int_{e^{-u}}^{1} \phi(s) f^{* *}(s) \frac{d s}{s} .
$$

This theorem allows us to omit the last supremum and to get the inequality

$$
\begin{aligned}
\|f\|_{\left(\Lambda_{\phi}, A_{\phi, \mathbf{b}}\right)_{D}^{K} w} & \lesssim\left\|\mathbf{b}\left(e^{-u}\right) \int_{e^{-u}}^{1} \phi(s) f^{* *}(s) \frac{d s}{s}\right\|_{E} \\
& =\left\|\mathbf{b}(t) \int_{t}^{1} \phi(s) f^{* *}(s) \frac{d s}{s}\right\|_{\widetilde{E}} \\
& =\|f\|_{A_{\phi, \mathbf{b}, E}}
\end{aligned}
$$

proving the embedding (4.6).

Now we proceed to studying interpolation properties of spaces $B_{\phi, \mathbf{b}, E}$ with respect to the Banach pair of Marcinkiewicz spaces $M_{\phi}$ and $M_{\phi, \mathbf{b}}$. First of all we should check that the space $B_{\phi, \mathbf{b}, E}$ is intermediate in this pair. Recall that $B_{\phi, \mathbf{b}, E} \subset L_{\phi, \mathbf{b}, E} \subset M_{\phi, \mathbf{b}}$, i.e., one of needed embeddings is fulfilled without any conditions. It turns out that the second embedding is not so universal. 
Proposition 4.3. Embedding $M_{\phi} \subset B_{\phi, \mathbf{b}, E}$ is equivalent to the condition $\mathbf{b}\left(e^{-u}\right) \in E$.

Proof. Let $f \in M_{\phi}$ with $\|f\|_{M_{\phi}}=1$, then $f^{* *}(t) \leq \frac{1}{\phi(t)}$ for all $t \in(0,1)$ and

$$
\begin{aligned}
\|f\|_{B_{\phi, \mathbf{b}, E}} & =\left\|\sup _{0<s<t} \phi(s) \mathbf{b}(s) f^{* *}(s)\right\|_{\widetilde{E}} \\
& \leq\left\|\sup _{0<s<t} \mathbf{b}(s)\right\|_{\widetilde{E}} \\
& =\|\mathbf{b}(t)\|_{\widetilde{E}} \\
& =\left\|\mathbf{b}\left(e^{-u}\right)\right\|_{E} .
\end{aligned}
$$

Hence, if $\mathbf{b}\left(e^{-u}\right) \in E$ then every $f \in M_{\phi}$ belongs to $B_{\phi, \mathbf{b}, E}$. Conversely, let $M_{\phi} \subset B_{\phi, \mathbf{b}, E}$. Since $\rho_{\phi}<1$, the space $M_{\phi}$ has an equivalent quasinorm $\sup _{t} \phi(t) f^{*}(t)$ and it is easy to see that $1 / \phi(t) \in M_{\phi}$. Therefore $1 / \phi(t) \in B_{\phi, \mathbf{b}, E}$ and

$$
\begin{aligned}
\left\|\frac{1}{\phi(t)}\right\|_{B_{\phi, \mathbf{b}, E}} & \sim\left\|\sup _{0<s<t} \phi(s) \mathbf{b}(s)\left(\frac{1}{\phi(s)}\right)^{*}\right\|_{\widetilde{E}} \\
& \sim\|\mathbf{b}(t)\|_{\widetilde{E}} \\
& =\left\|\mathbf{b}\left(e^{-u}\right)\right\|_{E}<\infty .
\end{aligned}
$$

The condition $\mathbf{b}\left(e^{-u}\right) \in E$ from the last proposition is the same as in Lemma 3.1. However, its role here is less crucial than previously, since the space $B_{\phi, \mathbf{b}, E}$ may be considered as nontrivial even for $\mathbf{b}\left(e^{-u}\right) \notin E$, while the space $A_{\phi, \mathbf{b}, E}$ in this case does not exist (contains only zero-function).

The basic interpolation property of the $B$-type spaces may be characterized by the following assertion.

Theorem 4.4. Let a r.i. space $D$ be defined by $\|f\|_{D}=\|F\|_{E}$ where $F(u)=f\left(\frac{1}{\mathbf{b}\left(e^{-u}\right)}\right), 0<u<\infty$. Then

$$
B_{\phi, \mathbf{b}, E} \supseteq\left(M_{\phi}, M_{\phi, \mathbf{b}}\right)_{D^{w}}^{K}, \quad \text { with } w(u)=\frac{1}{u}
$$

Proof. It is easy to show that, for all $u \in(0, \infty)$,

$$
K\left(u, f, M_{\phi}, M_{\phi, \mathbf{b}}\right) \sim\|f\|_{M_{\phi_{u}}},
$$

where

$$
\phi_{u}(s)=\phi(s) \min (1, u \mathbf{b}(s)) .
$$


Hence

$$
\begin{aligned}
\|f\|_{\left(M_{\phi}, M_{\phi, \mathbf{b}}\right)_{D}^{K} w} & =\left\|\frac{1}{u} K\left(u, f, M_{\phi}, M_{\phi, \mathbf{b}}\right)\right\|_{D} \\
& \sim\left\|\sup _{0<s<1} \min \left(\frac{1}{u}, \mathbf{b}(s)\right) \cdot \phi(s) f^{* *}(s)\right\|_{D} \\
& =\left\|\sup _{0<s<1} \min \left(\mathbf{b}\left(e^{-u}\right), \mathbf{b}(s)\right) \cdot \phi(s) f^{* *}(s)\right\|_{E} \\
& =\left\|\sup _{0<s<1} \min (\mathbf{b}(t), \mathbf{b}(s)) \cdot \phi(s) f^{* *}(s)\right\|_{\widetilde{E}} .
\end{aligned}
$$

Since the function $\mathbf{b}$ is increasing, we obtain that

$$
\|f\|_{\left(M_{\phi}, M_{\phi, \mathbf{b}}\right)_{D}^{K}} \geq\left\|\sup _{0<s<t} \mathbf{b}(s) \phi(s) f^{* *}(s)\right\|_{\widetilde{E}},
$$

which proves (4.7).

\section{The fundamental functions of $A$ and $B$ spaces}

In this section we will assume that the r.i. space $E$ and the function B (and thus also b) are connected by the condition $\rho_{E}<\pi_{\mathbf{B}}$. Due to Lemma 2.1 this condition is more restrictive than the necessary condition $\mathbf{b}\left(e^{-u}\right) \in E$, but not so much - e.g., when $E=L_{r}$ and $\mathbf{b}(t)=\left(\ln \frac{e}{t}\right)^{\alpha-1}$, both conditions are equivalent to $\frac{1}{r}<1-\alpha$ (the condition that appears in Bennett-Rudnick's results from [3]).

We start with studying the fundamental function of $A$-type spaces. For an estimation of $\varphi_{A_{\phi, \mathbf{b}, E}}$, we use the equivalent quasinorm in this space. Then, for all $\lambda \in(0,1)$,

$$
\begin{aligned}
\varphi_{A_{\phi, \mathbf{b}, E}}(\lambda) & \sim\left\|\mathbf{b}(t) \int_{t}^{1} \phi(s) \chi_{(0, \lambda)}(s) \frac{d s}{s}\right\|_{\widetilde{E}} \\
& =\left\|\chi_{(0, \lambda)}(t) \mathbf{b}(t) \int_{t}^{\lambda} \phi(s) \frac{d s}{s}\right\|_{\widetilde{E}} \\
& \lesssim \phi(\lambda)\left\|\mathbf{b}(t) \chi_{(0, \lambda)}(t)\right\|_{\widetilde{E}} .
\end{aligned}
$$

Using the fact that $\left\|f(u) \chi_{(a, \infty)}(u)\right\|_{E}=\|f(u+a)\|_{E}$ for all $a \in(0, \infty)$ and any r.i. $E$, we get

$\left\|\mathbf{b}(t) \chi_{(0, \lambda)}(t)\right\|_{\widetilde{E}}=\left\|\mathbf{b}\left(e^{-u}\right) \chi_{\left(\ln \frac{1}{\lambda}, \infty\right)}(u)\right\|_{E}=\left\|\mathbf{b}\left(e^{-u+\ln \lambda}\right)\right\|_{E}=\left\|\mathbf{b}\left(\lambda e^{-u}\right)\right\|_{E}$.

Hence, helping by Lemma 2.1, we obtain that

$$
\varphi_{A_{\phi, \mathbf{b}, E}}(\lambda) \lesssim \phi(\lambda) \mathbf{b}(\lambda) \varphi_{E}\left(\ln \frac{e}{\lambda}\right), \quad \text { for all } \lambda \in(0,1) .
$$


On the other hand,

$$
\begin{aligned}
\varphi_{A_{\phi, \mathbf{b}, E}}(\lambda) & \sim\left\|\chi_{(0, \lambda)}(t) \mathbf{b}(t) \int_{t}^{\lambda} \phi(s) \frac{d s}{s}\right\|_{\widetilde{E}} \\
& \geq\left\|\chi_{\left(0, \frac{\lambda}{2}\right)}(t) \mathbf{b}(t) \int_{\frac{\lambda}{2}}^{\lambda} \phi(s) \frac{d s}{s}\right\|_{\widetilde{E}} .
\end{aligned}
$$

Since $\phi(t) / t$ is decreasing, we get

$$
\int_{\frac{\lambda}{2}}^{\lambda} \phi(s) \frac{d s}{s} \geq \frac{\phi(\lambda)}{\lambda}\left(\lambda-\frac{\lambda}{2}\right) \gtrsim \phi(\lambda),
$$

and then, using (5.1), that $\mathbf{b}(t) / t$ is decreasing and inequality (2.2) from Lemma 2.1, we obtain

$$
\begin{aligned}
\varphi_{A_{\phi, \mathbf{b}, E}}(\lambda) & \gtrsim \phi(\lambda)\left\|\mathbf{b}(t) \chi_{\left(0, \frac{\lambda}{2}\right)}(t)\right\|_{\widetilde{E}} \\
& =\phi(\lambda)\left\|\mathbf{b}\left(\frac{\lambda}{2} e^{-u}\right)\right\|_{E} \\
& \gtrsim \phi(\lambda)\left\|\mathbf{b}\left(\lambda e^{-u}\right)\right\|_{E} \\
& \gtrsim \phi(\lambda) \mathbf{b}(\lambda) \varphi_{E}\left(\ln \frac{e}{\lambda}\right), \quad \text { for all } \lambda \in(0,1) .
\end{aligned}
$$

Summing up, we arrive at the final estimate

$$
\varphi_{A_{\phi, \mathbf{b}, E}}(\lambda) \sim \phi(\lambda) \mathbf{b}(\lambda) \varphi_{E}\left(\ln \frac{e}{\lambda}\right), \quad \text { for all } \lambda \in(0,1) .
$$

Remark 5.1. If we assume only the necessary condition $\mathbf{b}\left(e^{-u}\right) \in E$, then with the help of the inequality

$$
\begin{aligned}
\left\|\mathbf{b}\left(\lambda e^{-u}\right)\right\|_{E} & =\left\|\mathbf{B}\left(\frac{1}{u+\ln \frac{e}{\lambda}}\right)\right\|_{E} \\
& =\left\|\mathbf{B}\left(\frac{1}{\ln \frac{e}{\lambda}\left(1+\frac{u}{\ln \frac{e}{\lambda}}\right)}\right)\right\|_{E} \\
& \leq m_{\mathbf{B}}\left(\frac{1}{\ln \frac{e}{\lambda}}\right)\left\|\mathbf{B}\left(\frac{1}{1+\frac{u}{\ln \frac{e}{\lambda}}}\right)\right\|_{E} \\
& \leq m_{\mathbf{B}}\left(\frac{1}{\ln \frac{e}{\lambda}}\right) d_{E}\left(\ln \frac{e}{\lambda}\right)\left\|\mathbf{B}\left(\frac{1}{1+u}\right)\right\|_{E} \\
& =m_{\mathbf{B}}\left(\frac{1}{\ln \frac{e}{\lambda}}\right) d_{E}\left(\ln \frac{e}{\lambda}\right)\left\|\mathbf{b}\left(e^{-u}\right)\right\|_{E}
\end{aligned}
$$


we obtain the following estimate

$$
\phi(\lambda) \mathbf{b}(\lambda) \varphi_{E}\left(\ln \frac{e}{\lambda}\right) \lesssim \varphi_{A_{\phi, \mathbf{b}, E}}(\lambda) \lesssim \phi(\lambda) m_{\mathbf{B}}\left(\frac{1}{\ln \frac{e}{\lambda}}\right) d_{E}\left(\ln \frac{e}{\lambda}\right),
$$

for all $\lambda \in(0,1)$. This estimate is weaker than (5.2).

Next we will compute the fundamental functions of $B$-type spaces, using as before the equivalent quasi-norm in $B_{\phi, \mathbf{b}, E}$. At the first step we obtain that, for all $\lambda \in(0,1)$,

$$
\begin{aligned}
\varphi_{B_{\phi, \mathbf{b}, E}}(\lambda) & \sim\left\|\sup _{0<s<t} \phi(s) \mathbf{b}(s) \chi_{(0, \lambda)}(s)\right\|_{\widetilde{E}} \\
& \geq\left\|\chi_{\left(\frac{\lambda}{e}, 1\right)}(t) \sup _{0<s<\frac{\lambda}{e}} \phi(s) \mathbf{b}(s)\right\|_{\widetilde{E}} \\
& =\phi\left(\frac{\lambda}{e}\right) \mathbf{b}\left(\frac{\lambda}{e}\right)\left\|\chi_{\left(\frac{\lambda}{e}, 1\right)}(t)\right\|_{\widetilde{E}} \\
& \sim \phi(\lambda) \mathbf{b}(\lambda)\left\|\chi_{\left(0, \ln \frac{e}{\lambda}\right)}(u)\right\|_{E},
\end{aligned}
$$

where we took in account that the functions $\phi$ and $\mathbf{b}$ are increasing and the functions $\phi(t) / t$ and $\mathbf{b}(t) / t$ are decreasing. Thus we get the estimate from below

$$
\varphi_{B_{\phi, \mathbf{b}, E}}(\lambda) \gtrsim \phi(\lambda) \mathbf{b}(\lambda) \varphi_{E}\left(\ln \frac{e}{\lambda}\right), \quad \text { for all } \lambda \in(0,1) .
$$

For the estimate from above, we shall use the embedding $B_{\phi, \mathbf{b}, E} \supseteq$ $\left(M_{\phi}, M_{\phi, \mathbf{b}}\right)_{D^{w}}^{K}$, proved in Theorem 4.4, that gives

$$
\begin{aligned}
\varphi_{B_{\phi, \mathbf{b}, E}}(\lambda) & \lesssim\left\|\chi_{(0, \lambda)}(t)\right\|_{\left(M_{\phi}, M_{\phi, \mathbf{b}}\right)_{D}^{K}} \\
& =\left\|\frac{1}{u} K\left(u, \chi_{(0, \lambda)}(t), M_{\phi}, M_{\phi, \mathbf{b}}\right)\right\|_{D} .
\end{aligned}
$$

Moreover,

$$
\begin{aligned}
K\left(u, \chi_{(0, \lambda)}(t), M_{\phi}, M_{\phi, \mathbf{b}}\right) & \sim\left\|\chi_{(0, \lambda)}\right\|_{M_{\phi_{u}}} \\
& =\varphi_{M_{\phi_{u}}}(\lambda) \sim \phi_{u}(\lambda) \\
& =\phi(\lambda) \min (1, u \mathbf{b}(\lambda)),
\end{aligned}
$$

hence

$$
\begin{aligned}
\varphi_{B_{\phi, \mathbf{b}, E}}(\lambda) & \lesssim \phi(\lambda)\left\|\min \left(\frac{1}{u}, \mathbf{b}(\lambda)\right)\right\|_{D} \\
& =\phi(\lambda)\left\|\min \left(\mathbf{b}\left(e^{-u}\right), \mathbf{b}(\lambda)\right)\right\|_{E} \\
& =\phi(\lambda) \mathbf{b}(\lambda)\left\|\min \left(1, \frac{\mathbf{b}\left(e^{-u}\right)}{\mathbf{b}(\lambda)}\right)\right\|_{E}
\end{aligned}
$$


Furthermore, using (5.1) and inequality $\varphi_{E}\left(\ln \frac{1}{\lambda}\right) \leq \varphi_{E}\left(\ln \frac{e}{\lambda}\right)$, we get

$$
\begin{aligned}
\left\|\min \left(1, \frac{\mathbf{b}\left(e^{-u}\right)}{\mathbf{b}(\lambda)}\right)\right\|_{E} & \leq\left\|\chi_{\left(0, \ln \frac{1}{\lambda}\right)}(u)\right\|_{E}+\frac{1}{\mathbf{b}(\lambda)}\left\|\mathbf{b}\left(e^{-u}\right) \chi_{\left(\ln \frac{1}{\lambda}, \infty\right)}(u)\right\|_{E} \\
& \leq \varphi_{E}\left(\ln \frac{e}{\lambda}\right)+\frac{1}{\mathbf{b}(\lambda)}\left\|\mathbf{b}\left(\lambda e^{-u}\right)\right\|_{E} .
\end{aligned}
$$

Thus we obtain that

$$
\varphi_{B_{\phi, \mathbf{b}, E}}(\lambda) \lesssim \phi(\lambda) \mathbf{b}(\lambda) \varphi_{E}\left(\ln \frac{e}{\lambda}\right)+\phi(\lambda)\left\|\mathbf{b}\left(\lambda e^{-u}\right)\right\|_{E}, \quad \text { for all } \lambda \in(0,1) .
$$

Applying as before Lemma 2.1, we can see that the second summand in the last inequality is no greater than the first one, which, together with (5.3), gives the final estimate

$$
\varphi_{B_{\phi, \mathbf{b}, E}}(\lambda) \sim \phi(\lambda) \mathbf{b}(\lambda) \varphi_{E}\left(\ln \frac{e}{\lambda}\right), \quad \text { for all } \lambda \in(0,1) .
$$

Remark 5.2. As in the case of the space $A_{\phi, \mathbf{b}, E}$, if we assume only the necessary condition $\mathbf{b}\left(e^{-u}\right) \in E$, we obtain the estimate

$$
\phi(\lambda) \mathbf{b}(\lambda) \varphi_{E}\left(\ln \frac{e}{\lambda}\right) \lesssim \varphi_{B_{\phi, \mathbf{b}, E}}(\lambda) \lesssim \phi(\lambda) m_{\mathbf{B}}\left(\frac{1}{\ln \frac{e}{\lambda}}\right) d_{E}\left(\ln \frac{e}{\lambda}\right),
$$

for all $\lambda \in(0,1)$.

Notice that, for one and the same set of parameters $\phi, \mathbf{b}, E$, the estimates (5.2) and (5.4) for fundamental functions of spaces $A_{\phi, \mathbf{b}, E}$ and $B_{\phi, \mathbf{b}, E}$ are identical, while the fundamental function of the ultrasymmetric space $L_{\phi, \mathbf{b}, E}$ with the same set of parameters is $\phi(\lambda) \mathbf{b}(\lambda)$ independently of $E$. This fact ensures once again that the spaces $A_{\phi, \mathbf{b}, E}$ and $B_{\phi, \mathbf{b}, E}$ are different from $L_{\phi, \mathbf{b}, E}$ if $\lim _{t \rightarrow \infty} \varphi_{E}(t)=\infty$.

\section{Embeddings between spaces of $A$ type and ultrasymmetric spaces}

We know already that any space $A_{\phi, \mathbf{b}, E}$ is not larger than the ultrasymmetric space $L_{\phi, \mathbf{b}, E}$ (see Remark 3.3). In this section we will show that, under condition $\rho_{E}<\pi_{\mathbf{B}}$, the space $A_{\phi, \mathbf{b}, E}$ lies between ultrasymmetric spaces $L_{\phi, \mathbf{b}, E}$ and $L_{\phi, \mathbf{b}} \ln \frac{e}{t}, E$.

Lemma 6.1. Let a linear operator

$$
P g(u)=\mathbf{b}\left(e^{-u}\right) \int_{0}^{u} \frac{g(v)}{\mathbf{b}\left(e^{-v}\right)(1+v)} d v, \quad 0<u<\infty
$$


be bounded on some r.i. space $E$. Then $L_{\phi, \mathbf{b} \ln \frac{e}{t}, E} \subset A_{\phi, \mathbf{b}, E}$.

Proof. Let $f \in L_{\phi, \mathbf{b} \ln \frac{e}{t}, E}$. Set $g(u)=\mathbf{b}\left(e^{-u}\right)(1+u) \phi\left(e^{-u}\right) f^{* *}\left(e^{-u}\right)$. The boundedness of $P$ implies that

$$
\begin{aligned}
\|P g\|_{E} & =\left\|\mathbf{b}\left(e^{-u}\right) \int_{0}^{u} \phi\left(e^{-v}\right) f^{* *}\left(e^{-v}\right) d v\right\|_{E} \lesssim\|g\|_{E} \\
& =\left\|\mathbf{b}\left(e^{-u}\right)(1+u) \phi\left(e^{-u}\right) f^{* *}\left(e^{-u}\right)\right\|_{E} .
\end{aligned}
$$

Observing that

$$
\begin{aligned}
\|P g\|_{E} & =\left\|\mathbf{b}(t) \int_{0}^{\ln \frac{1}{t}} \phi\left(e^{-v}\right) f^{* *}\left(e^{-v}\right) d v\right\|_{\widetilde{E}} \\
& =\left\|\mathbf{b}(t) \int_{t}^{1} \phi(s) f^{* *}(s) \frac{d s}{s}\right\|_{\widetilde{E}} \\
& =\|f\|_{A_{\phi, \mathbf{b}, E}}
\end{aligned}
$$

and

$$
\|g\|_{E}=\left\|\mathbf{b}(t)\left(\ln \frac{e}{t}\right) \phi(t) f^{* *}(t)\right\|_{\widetilde{E}} \sim\|f\|_{L_{\phi, \mathbf{b} \ln \frac{e}{t}, E}},
$$

we finish the proof.

Lemma 6.2. The operator (6.1) is bounded in $L_{\infty}$ whenever $\pi_{\mathbf{B}}>0$.

Proof. Using Lemma 2.2, we obtain that

$$
|P g(u)| \leq\left(\sup _{0<v<u}|g(v)|\right) \mathbf{b}\left(e^{-u}\right) \int_{0}^{u} \frac{1}{\mathbf{b}\left(e^{-v}\right)(1+v)} d v \lesssim \sup _{0<v<u}|g(v)|
$$

for all $u>0$ and thus

$$
\|P g\|_{L_{\infty}} \lesssim\|g\|_{L_{\infty}} .
$$

Now we are able to prove the embedding $L_{\phi, \mathbf{b} \ln \frac{e}{t}, E} \subset A_{\phi, \mathbf{b}, E}$ under the condition $\rho_{E}<\pi_{\mathbf{B}}$. Moreover, this condition can be mollified to $\mathbf{b}\left(e^{-u}\right) \in E$ in the case when $\pi_{\mathbf{B}}>1$.

Theorem 6.3. Let $\pi_{\mathbf{B}}>1$. Then the embedding $L_{\phi, \mathbf{b} \ln \frac{e}{t}, E} \subset A_{\phi, \mathbf{b}, E}$ holds for every r.i. space $E$ such that $\mathbf{b}\left(e^{-u}\right) \in E$.

Proof. Let us show that the operator (6.1) is bounded in $L_{1}$. Indeed,

$$
\begin{aligned}
\|P g\|_{L_{1}} & \leq \int_{0}^{\infty} \mathbf{b}\left(e^{-u}\right)\left(\int_{0}^{u} \frac{|g(v)|}{\mathbf{b}\left(e^{-v}\right)(1+v)} d v\right) d u \\
& =\int_{0}^{\infty} \frac{|g(v)|}{\mathbf{b}\left(e^{-v}\right)(1+v)}\left(\int_{v}^{\infty} \mathbf{b}\left(e^{-u}\right) d u\right) d v .
\end{aligned}
$$

Using Lemma 2.3, we obtain that the operator $P$ is bounded in $L_{1}$. This fact together with Lemma 6.2 gives us that the operator $P$ is bounded in 
any interpolation space for the Banach pair $\left(L_{1}, L_{\infty}\right)$, that is, in any r.i. space $E$. And then Lemma 6.1 ensures the desired embedding.

Theorem 6.4. Let $0<\pi_{\mathbf{B}} \leq 1$. Then the embedding $L_{\phi, \mathbf{b} \ln \frac{e}{t}, E} \subset$ $A_{\phi, \mathbf{b}, E}$ holds for every r.i. space $E$ such that $\rho_{E}<\pi_{\mathbf{B}}$.

Proof. Let us take a number $p$ such that $\rho_{E}<1 / p<\pi_{\mathbf{B}}$. Then the function $B(u) u^{-1 / p}$ is almost increasing and thus the function $\mathbf{b}\left(e^{-v}\right)(1+v)^{1 / p}$ is almost decreasing. Therefore

$$
\begin{aligned}
\mathbf{b}\left(e^{-u}\right) \int_{0}^{u} \frac{g(v)}{\mathbf{b}\left(e^{-v}\right)(1+v)} d v & \lesssim \frac{1}{(1+u)^{1 / p}} \int_{0}^{u} \frac{g(v)}{(1+v)^{1-1 / p}} d v \\
& \leq \frac{1}{u^{1 / p}} \int_{0}^{u} \frac{g(v)}{v^{1-1 / p}} d v
\end{aligned}
$$

It is known (see, e.g., [9, p. 245]) that the operator

$$
H g(u)=\frac{1}{u^{1 / p}} \int_{0}^{u} \frac{g(v)}{v^{1-1 / p}} d v
$$

is bounded in any $L_{q}$ with $q>p$, for instance, in $L_{q}$ with $\rho_{E}<1 / q<1 / p$. Consequently, we obtain that the operator (6.1) is bounded in the same $L_{q}$. Since by Lemma 6.2 it is also bounded in $L_{\infty}$, this gives that $P$ is bounded in any $E$ with $\rho_{E}<1 / q$. But the numbers $p, q$ are arbitrary, so we obtain the same assertion under condition $\rho_{E}<\pi_{\mathbf{B}}$ as desired.

Summing up, we may conclude that, for any r.i. $E$ such that $\mathbf{b}\left(e^{-u}\right) \in E$ if $\pi_{\mathbf{B}}>1$ or $\rho_{E}<\pi_{\mathbf{B}}$ if $0<\pi_{\mathbf{B}} \leq 1$, the $A$-type and the ultrasymmetric spaces are connected by the relation

$$
L_{\phi, \mathbf{b} \ln \frac{e}{t}, E} \subset A_{\phi, \mathbf{b}, E} \subset L_{\phi, \mathbf{b}, E} .
$$

Moreover, by comparison of corresponding fundamental functions, we may assert that these embeddings are strict whenever the function $\varphi_{E}(t)$ is not equivalent to 1 or to $t$. Taking $f(t)=\frac{1}{\phi(t)}$, one can readily check that, for $\varphi_{E}(t) \sim 1$, the spaces $A_{\phi, \mathbf{b}, E}$ and $L_{\phi, \mathbf{b}, E}$ are different too. It can be shown that in the remaining case $\varphi_{E}(t) \sim t$ the space $A_{\phi, \mathbf{b}, E}$ coincides with $L_{\phi, \mathbf{b} \ln \frac{e}{t}, E}$. Let us prove this assertion for the principal case $E=L_{1}$ (recall that $\rho_{L_{1}}=1$ ).

Proposition 6.5. If $\pi_{\mathbf{B}}>1$ then $A_{\phi, \mathbf{b}, L_{1}}=L_{\phi, \mathbf{b} \ln \frac{e}{t}, L_{1}}$.

Proof. By a mere change of integrals we have

$$
\|f\|_{A_{\phi, \mathbf{b}, L_{1}}}=\int_{0}^{1} \mathbf{b}(t)\left(\int_{t}^{1} \phi(s) f^{* *}(s) \frac{d s}{s}\right) \frac{d t}{t}
$$




$$
=\int_{0}^{1} \phi(s) f^{* *}(s)\left(\int_{0}^{s} \mathbf{b}(t) \frac{d t}{t}\right) \frac{d s}{s} .
$$

Changing variables and using Lemma 2.3, we get

$$
\int_{0}^{s} \mathbf{b}(t) \frac{d t}{t}=\int_{\ln \frac{1}{s}}^{\infty} \mathbf{b}\left(e^{-v}\right) d v \sim \mathbf{b}(s) \ln \frac{e}{s} .
$$

Therefore

$$
\begin{aligned}
\|f\|_{A_{\phi, \mathbf{b}, L_{1}}} & \sim \int_{0}^{1} \phi(s) \mathbf{b}(s)\left(\ln \frac{e}{s}\right) f^{* *}(s) \frac{d s}{s} \\
& \sim\|f\|_{L_{\phi, \mathbf{b} \ln \frac{e}{t}, L_{1}}},
\end{aligned}
$$

and the assertion is proved.

\section{Weak type interpolation theorems}

The weak type interpolation, that has its origins in the classical Marcinkiewicz interpolation theorem, relates to interpolation properties of operators $T$ which map a pair of Lorentz spaces into a pair of Marcinkiewicz spaces. In accordance with modern terminology, we say that a (quasi)linear operator $T$ is of weak type $(\phi, \psi)$ if $T: \Lambda_{\phi} \rightarrow M_{\psi}$. We denote by $W\left(\phi_{0}, \psi_{0} ; \phi_{1}, \psi_{1}\right)$ the set of all (quasi)linear operators which are of weak type $\left(\phi_{0}, \psi_{0}\right)$ and also of weak type $\left(\phi_{1}, \psi_{1}\right)$. For obtaining reasonably informative results, we assume additionally that the ratios $\phi_{0} / \phi_{1}$ and $\psi_{0} / \psi_{1}$ are also monotone with sufficiently rapid growth.

In the present paper we study only spaces with underlying interval $(0,1)$, so that all parameter functions $\phi(t), \phi_{0}(t)$ etc. may be defined only for $t \in(0,1)$. But all they are quasiconcave and thus continuous, so we always may define $\phi(1)=\lim _{t \rightarrow 1} \phi(t)$. Moreover, when using general assertions from interpolation theory, we need sometimes the values of these functions for $t>1$. Recall (see Section 2 above) that we agreed to extend all parameter functions (when it is needed) to the whole interval $(0, \infty)$, setting $\phi(t)=\phi(1)$ for all $t>1$.

The weak type interpolation results for ultrasymmetric spaces, which are not "too close" to the endpoint spaces, were stated in [13, Theorem 5.1], where it was shown that, for every quasi-power function $\theta(t)$, any operator $T \in W\left(\phi_{0}, \psi_{0} ; \phi_{1}, \psi_{1}\right)$ is bounded from $L_{\phi, E}$ to $L_{\psi, E}$ with

$$
\phi=\phi_{1} \theta\left(\frac{\phi_{0}}{\phi_{1}}\right), \quad \psi=\psi_{1} \theta\left(\frac{\psi_{0}}{\psi_{1}}\right) .
$$

The case of spaces, which are "close" to the endpoint spaces, was studied in the paper [15] for power functions $\phi_{i}, \psi_{i}, i=0,1$. Extension of these 
results to arbitrary quasi-power functions is only a technical problem; one of extended results was given above (Theorem 1.1).

In this section we will show that, using spaces of $A$ and $B$ types, we can get interpolation results which are stronger than that of Theorem 1.1. In order to do this we have to accomplish the interpolation in two stages.

Lemma 7.1. Let $\phi_{0}, \psi_{0}$ be two quasiconcave functions such that $\pi_{\phi_{0}}>0$ and $\pi_{\psi_{0}}>0$, let $\phi_{1}$ and $\psi_{1}$ be two quasi-power functions and let $\pi_{\phi_{0} / \phi_{1}}>0$ and $\pi_{\psi_{0} / \psi_{1}}>0$. If $T \in W\left(\phi_{0}, \psi_{0} ; \phi_{1}, \psi_{1}\right)$ then $T: A_{\phi_{1}, \mathbf{b}} \rightarrow M_{\psi_{1}, \mathbf{b}}$, where $A_{\phi_{1}, \mathbf{b}}$ stands for the space $A_{\phi_{1}, \mathbf{b}, L_{\infty}}$ (cf. (4.1)).

Proof. Let us consider the interpolation functor $\mathbf{M}_{\mathbf{b}}$, defined for arbitrary Banach pair $\left(A_{0}, A_{1}\right)$ as the space $\mathbf{M}_{\mathbf{b}}\left(A_{0}, A_{1}\right)$ consisting of all $f \in A_{0}+A_{1}$ with finite norm

$$
\|f\|_{M_{\mathbf{b}}\left(A_{0}, A_{1}\right)}=\sup _{0<t<1} \frac{\mathbf{b}(t)}{t} K\left(t, f, A_{0}, A_{1}\right) .
$$

For the Banach pair $\left(M_{\psi_{0}}, M_{\psi_{1}}\right)$, this functor gives a space $\mathbf{M}_{\mathbf{b}}\left(M_{\psi_{0}}, M_{\psi_{1}}\right)=$ $M_{\psi}$, where $\psi(t)=\psi_{1}(t) \mathbf{b}\left(\psi_{0}(t) / \psi_{1}(t)\right)$. Set $\Psi(t)=\psi_{0}(t) / \psi_{1}(t)$. Since $\psi_{0}(t) / t$ is decreasing for all $t \in(0,1)$ and $\psi_{1}(t)$ is increasing, the function $\Psi(t) / t$ is decreasing. Hence $t \Psi(1) \leq \Psi(t)$ and $\mathbf{b}(t) \lesssim \mathbf{b}(\Psi(t))$. Consequently $\psi_{1}(t) \mathbf{b}(t) \lesssim \psi(t)$ and thus $\mathbf{M}_{\mathbf{b}}\left(M_{\psi_{0}}, M_{\psi_{1}}\right) \subset M_{\psi_{1}, \mathbf{b}}$.

Let now $T \in W\left(\phi_{0}, \psi_{0} ; \phi_{1}, \psi_{1}\right)$. As for any interpolation functor, we have that $T: \mathbf{M}_{\mathbf{b}}\left(\Lambda_{\phi_{0}}, \Lambda_{\phi_{1}}\right) \rightarrow \mathbf{M}_{\mathbf{b}}\left(M_{\psi_{0}}, M_{\psi_{1}}\right)$, hence in order to get the desired interpolation result it is enough to prove the embedding $A_{\phi_{1}, \mathbf{b}} \subset \mathbf{M}_{\mathbf{b}}\left(\Lambda_{\phi_{0}}, \Lambda_{\phi_{1}}\right)$.

Set $\Phi(t)=\phi_{0}(t) / \phi_{1}(t)$. Observe that $0<\pi_{\Phi} \leq \rho_{\Phi} \leq \rho_{\phi_{0}}-\pi_{\phi_{1}}<1$, and hence $\Phi$ is a quasi-power function. Passing, if necessary, to equivalent parameter functions, we obtain that $\Phi$ satisfies

$$
0<\widetilde{\pi}_{\Phi} \leq \frac{t \Phi^{\prime}(t)}{\Phi(t)} \leq \widetilde{\rho}_{\Phi}<1 \quad \text { for all } t>0
$$

with some numbers $\tilde{\pi}_{\Phi}<\pi_{\Phi}$ and $\tilde{\rho}_{\Phi}>\rho_{\Phi}$. These inequalities allow us to apply the generalized Holmstedt's formula from [11] and to obtain the $K$-functional for the pair $\left(\Lambda_{\phi_{0}}, \Lambda_{\phi_{1}}\right)$ in the form

$$
K\left(t, f, \Lambda_{\phi_{0}}, \Lambda_{\phi_{1}}\right) \sim \int_{0}^{\eta(t)} \frac{K\left(s, f, L_{1}, L_{\infty}\right)}{s / \phi_{0}(s)} \frac{d s}{s}+t \int_{\eta(t)}^{\infty} \frac{K\left(s, f, L_{1}, L_{\infty}\right)}{s / \phi_{1}(s)} \frac{d s}{s}
$$

where $\eta(t)$ is the inverse function to $\Phi(t)$. In result, the norm in $\mathbf{M}_{\mathbf{b}}\left(\Lambda_{\phi_{0}}, \Lambda_{\phi_{1}}\right)$ is no greater than

$$
\sup _{0<t<1} \frac{\mathbf{b}(t)}{t} \int_{0}^{\eta(t)} \phi_{0}(s) f^{* *}(s) \frac{d s}{s}+\sup _{0<t<1} \mathbf{b}(t) \int_{\eta(t)}^{\infty} \phi_{1}(s) f^{* *}(s) \frac{d s}{s}
$$


and we only have to compare (7.2) with $\|f\|_{A_{\phi_{1}, \mathrm{~b}}}$.

In order to estimate the first supremum we may assume (due to homogeneity) that $\|f\|_{A_{\phi_{1}, \mathbf{b}}}=1$. Since $\mathbf{b}(t) / t$ is decreasing, we get from (4.1)

$$
\int_{t / 2}^{t} \phi_{1}(s) f^{* *}(s) \frac{d s}{s} \leq \int_{t / 2}^{1} \phi_{1}(s) f^{* *}(s) \frac{d s}{s} \leq \frac{1}{\mathbf{b}(t / 2)} \lesssim \frac{1}{\mathbf{b}(t)} .
$$

On the other hand,

$$
\int_{t / 2}^{t} \phi_{1}(s) f^{* *}(s) \frac{d s}{s} \geq f^{* *}(t) \int_{t / 2}^{t} \frac{\phi_{1}(s)}{s} d s \geq f^{* *}(t) \frac{\phi_{1}(t)}{t}\left(t-\frac{t}{2}\right) \sim f^{* *}(t) \phi_{1}(t),
$$

therefore

$$
f^{* *}(t) \lesssim \frac{1}{\phi_{1}(t) \mathbf{b}(t)}
$$

Furthermore, it is easy to obtain from (7.1) that

$$
1<\frac{1}{\widetilde{\rho}_{\Phi}} \leq \frac{t \eta^{\prime}(t)}{\eta(t)} \leq \frac{1}{\widetilde{\pi}_{\Phi}}<\infty
$$

which implies that $\eta(t) \gtrsim t^{1 / \widetilde{\pi}_{\Phi}}$ for $t \in(0,1)$, whence $\mathbf{b}(\eta(t)) \gtrsim \mathbf{b}\left(t^{1 / \widetilde{\pi}_{\Phi}}\right)$ due to the function $\mathbf{b}(t)$ being increasing. At the same time, $\mathbf{b}\left(t^{2}\right) \sim \mathbf{b}(t)$ and thus $\mathbf{b}\left(t^{\alpha}\right) \sim \mathbf{b}(t)$ for any $\alpha>0$. Using these facts, we obtain that, for any $t \in(0,1)$,

$$
\begin{aligned}
\int_{0}^{\eta(t)} \phi_{0}(s) f^{* *}(s) \frac{d s}{s} & \lesssim \int_{0}^{\eta(t)} \frac{\phi_{0}(s)}{\phi_{1}(s)} \frac{1}{\mathbf{b}(s)} \frac{d s}{s} \\
& =\int_{0}^{\eta(t)} \Phi(s) \frac{1}{\mathbf{b}(s)} \frac{d s}{s} \\
& \lesssim \int_{0}^{t} \frac{1}{\mathbf{b}(\eta(u))} d u \\
& \lesssim \int_{0}^{t} \frac{1}{\mathbf{b}(u)} d u
\end{aligned}
$$

Since the function $t / \mathbf{b}(t)$ is increasing, this implies that

$$
\begin{aligned}
\int_{0}^{t} \frac{1}{\mathbf{b}(u)} d u & =\int_{0}^{\sqrt{t}} \frac{2 s}{\mathbf{b}\left(s^{2}\right)} d s \\
& \sim \int_{0}^{\sqrt{t}} \frac{s}{\mathbf{b}(s)} d s \\
& \leq \frac{\sqrt{t}}{\mathbf{b}(\sqrt{t})} \cdot \sqrt{t} \sim \frac{t}{\mathbf{b}(t)}
\end{aligned}
$$


and hence

So we have proved that

$$
\int_{0}^{\eta(t)} \phi_{0}(s) f^{* *}(s) \frac{d s}{s} \lesssim \frac{t}{\mathbf{b}(t)}
$$

$$
\sup _{0<t<1} \frac{\mathbf{b}(t)}{t} \int_{0}^{\eta(t)} \phi_{0}(s) f^{* *}(s) \frac{d s}{s} \lesssim\|f\|_{A_{\phi_{1}, \mathbf{b}}} .
$$

In order to estimate the second supremum in (7.2) we observe that (passing to equivalent functions, if necessary) we always may assume that $\Phi(1)=1$. Since $\Phi(t) / t$ is decreasing, this gives immediately that $t \leq \Phi(t)$ for all $t \in(0,1)$ and then $\eta(t) \leq t<1$. Hence

$$
\begin{aligned}
\int_{\eta(t)}^{\infty} \phi_{1}(s) f^{* *}(s) \frac{d s}{s} & =\int_{\eta(t)}^{1} \phi_{1}(s) f^{* *}(s) \frac{d s}{s}+\int_{1}^{\infty} \phi_{1}(s) f^{* *}(s) \frac{d s}{s} \\
& =\int_{\eta(t)}^{1} \phi_{1}(s) f^{* *}(s) \frac{d s}{s}+f^{* *}(1) \phi_{1}(1) \int_{1}^{\infty} \frac{d s}{s^{2}} \\
& =\int_{\eta(t)}^{1} \phi_{1}(s) f^{* *}(s) \frac{d s}{s}+\|f\|_{L_{1}(0,1)} \phi_{1}(1) .
\end{aligned}
$$

Observe also that $A_{\phi_{1}, \mathbf{b}} \subset L_{1}$ as any r.i. space on $(0,1)$, i.e., $\|f\|_{L_{1}(0,1)} \lesssim$ $\|f\|_{A_{\phi_{1}, \mathbf{b}}}$. Moreover, using that $\mathbf{b}(t) \lesssim \mathbf{b}(\eta(t))$ for $t \in(0,1)$, we get

$$
\begin{aligned}
\sup _{0<t<1} \mathbf{b}(t) \int_{\eta(t)}^{1} \phi_{1}(s) f^{* *}(s) \frac{d s}{s} & \lesssim \sup _{0<t<1} \mathbf{b}(\eta(t)) \int_{\eta(t)}^{1} \phi_{1}(s) f^{* *}(s) \frac{d s}{s} \\
& \leq \sup _{0<u<1} \mathbf{b}(u) \int_{u}^{1} \phi_{1}(s) f^{* *}(s) \frac{d s}{s} \\
& =\|f\|_{A_{\phi_{1}, \mathbf{b}}} .
\end{aligned}
$$

Summing both estimates, we obtain that $\|f\|_{M_{\mathbf{b}}\left(\Lambda_{\phi_{0}}, \Lambda_{\phi_{1}}\right)} \lesssim\|f\|_{A_{\phi_{1}, \mathbf{b}}}$ and the proof is finished.

Now we are able to prove the main interpolation theorem of our paper.

Theorem 7.2. Let $\phi_{0}, \psi_{0}$ be two quasiconcave functions such that $\pi_{\phi_{0}}>0$ and $\pi_{\psi_{0}}>0$. Let $\phi_{1}$ and $\psi_{1}$ be two quasi-power functions and let $\pi_{\phi_{0} / \phi_{1}}>0$ and $\pi_{\psi_{0} / \psi_{1}}>0$. If $T \in W\left(\phi_{0}, \psi_{0} ; \phi_{1}, \psi_{1}\right)$, then $T: A_{\phi_{1}, \mathbf{b}, E} \rightarrow B_{\psi_{1}, \mathbf{b}, E}$ for any r.i. space $E$ containing the function $\mathbf{b}\left(e^{-u}\right)$.

Proof. We apply Lemma 7.1 in order to obtain that $T$ is bounded as an operator from $A_{\phi_{1}, \mathbf{b}}$ to $M_{\psi_{1}, \mathbf{b}}$. At the same time, $T: \Lambda_{\phi_{1}} \rightarrow M_{\psi_{1}}$. By the real interpolation we then obtain that

$$
T:\left(\Lambda_{\phi_{1}}, A_{\phi_{1}, \mathbf{b}}\right)_{D^{w}}^{K} \rightarrow\left(M_{\psi_{1}}, M_{\psi_{1}, \mathbf{b}}\right)_{D^{w}}^{K}
$$


for the same space $D$ and function $w$ as in Theorems 4.2 and 4.4. But by Theorem 4.2 we have that $A_{\phi_{1}, \mathbf{b}, E} \subseteq\left(\Lambda_{\phi_{1}}, A_{\phi_{1}, \mathbf{b}}\right)_{D^{w}}^{K}$ and by Theorem 4.4 we have that $B_{\psi_{1}, \mathbf{b}, E} \supseteq\left(M_{\psi_{1}}, M_{\psi_{1}, \mathbf{b}}\right)_{D^{w}}^{K}$, so that

$$
T: A_{\phi_{1}, \mathbf{b}, E} \rightarrow B_{\psi_{1}, \mathbf{b}, E}
$$

and the proof is finished.

We know that any space $B_{\psi_{1}, \mathbf{b}, E}$ is not larger than the space $L_{\psi_{1}, \mathbf{b}, E}$, therefore the result of the last theorem remains true if we replace the space $B_{\psi_{1}, \mathbf{b}, E}$ by the space $L_{\psi_{1}, \mathbf{b}, E}$ as a range space of interpolation. Similarly we know from Theorems 6.3 and 6.4 that, under some additional conditions, the space $L_{\phi_{1}, \mathbf{b} \ln \frac{e}{t}, E}$ is smaller than $A_{\phi_{1}, \mathbf{b}, E}$ and thus it may replace $A_{\phi_{1}, \mathbf{b}, E}$ as a domain space of interpolation. In other words, we can derive from the embeddings

$$
L_{\phi_{1}, \mathbf{b} \ln \frac{e}{t}, E} \subset A_{\phi_{1}, \mathbf{b}, E}, \quad B_{\psi_{1}, \mathbf{b}, E} \subset L_{\psi_{1}, \mathbf{b}, E}
$$

the following interpolation theorem, involving ultrasymmetric spaces.

Theorem 7.3. Let $\phi_{0}, \psi_{0}$ be two quasiconcave functions such that $\pi_{\phi_{0}}>0$ and $\pi_{\psi_{0}}>0$. Let $\phi_{1}$ and $\psi_{1}$ be two quasi-power functions and let $\pi_{\phi_{0} / \phi_{1}}>0$ and $\pi_{\psi_{0} / \psi_{1}}>0$. Let $E$ be any r.i. space on $(0, \infty)$ such that $\mathbf{b}\left(e^{-u}\right) \in E$ if $\pi_{\mathbf{B}}>1$ or $\rho_{E}<\pi_{\mathbf{B}}$ if $0<\pi_{\mathbf{B}} \leq 1$. Then, for any (quasi)linear operator $T \in W\left(\phi_{0}, \psi_{0} ; \phi_{1}, \psi_{1}\right)$, each of the following assertions is true:

(i) $T: A_{\phi_{1}, \mathbf{b}, E} \rightarrow L_{\psi_{1}, \mathbf{b}, E}$,

(ii) $T: L_{\phi_{1}, \mathbf{b} \ln \frac{e}{t}, E} \rightarrow B_{\psi_{1}, \mathbf{b}, E}$,

(iii) $T: L_{\phi_{1}, \mathbf{b} \ln \frac{e}{t}, E} \rightarrow L_{\psi_{1}, \mathbf{b}, E}$.

Acknowledgements. We are thankful to the referee for his/her valuable remarks and comments.

\section{References}

[1] D. W. Boyd, Indices of function spaces and their relationship to interpolation, Canad. J. Math., 21 (1969), 1245-1254.

[2] Ju. A. Brudnyi and N. Ja. Krugljak, Interpolation Functors and Interpolation spaces, Vol. I, North-Holland, Amsterdam, 1991.

[3] C. Bennett and K. Rudnick, On Lorentz-Zygmund spaces, Dissertationes Math., 175 (1980), 5-67.

[4] C. Bennett and R. Sharpley, Interpolation of Operators, Academic Press, Boston, 1988. 
[5] A. Calderón, Spaces between $L^{1}$ and $L^{\infty}$ and the theorem of Marcinkiewicz, Studia Math., 26 (1966), 273-299.

[6] M. Cwikel and E. Pustylnik, Weak type interpolation near "endpoint" spaces, J. Funct. Anal., 171 (2000), 235-277.

[7] D. E. Edmunds, P. Gurka and B. Opic, Double exponential integrability of convolution operators in generalized Lorentz-Zygmund spaces, Indiana Univ. Math. J., 44 (1995), 19-43.

[8] W. D. Evans, B. Opic and L. Pick, Interpolation of operators on scales of generalized Lorentz-Zygmund spaces, Math. Nachr., 182 (1996), 127181.

[9] G. H. Hardy, J. E. Littlewood and G. Pólya, Inequalities, Cambridge University Press, 1952.

[10] S. G. Kreǐn, Ju. I. Petunin and E. M. Semenov, Interpolation of Linear Operators, Translations of Math. Monographs, Vol. 54, Amer. Math. Soc., Providence, R.I., 1982.

[11] L. E. Persson, Interpolation with a parameter function, Math. Scand., 59 (1986), 199-222.

[12] E. Pustylnik, Optimal interpolation in spaces of Lorentz-Zygmund type, J. d'Analyse Math., 79 (1999), 113-157.

[13] E. Pustylnik, Ultrasymmetric spaces, J. London Math. Soc., 68 (2003), 165-182.

[14] E. Pustylnik, Weak type interpolation in quasinormed spaces of measurable functions, Function Spaces, Proc. of the Sixth Conf., Wroclaw", World Sci. Publ., 2003, 225-236.

[15] E. Pustylnik, Extreme cases of weak type interpolation, Rev. Mat. Iberoamericana, 21 (2005), 557-576.

Department of Mathematics

Technion-Israel Institute of Technology

Haifa 32000

Israel

(E-mail : evg@techunix.technion.ac.il)

Departamento de Matemática Aplicada

Facultad de Informática

Universidad de Murcia

Campus de Espinardo

30100 Espinardo (Murcia)

Spain

(E-mail : tmsignes@um.es)

(Received : December 2005) 


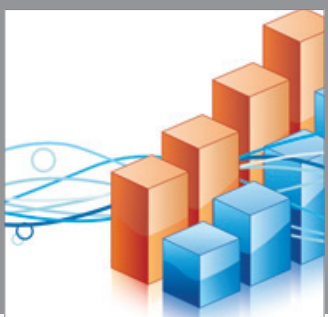

Advances in

Operations Research

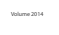

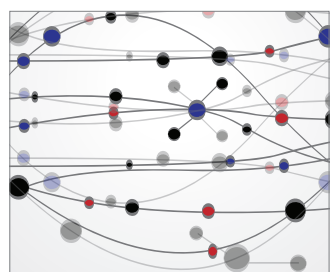

\section{The Scientific} World Journal
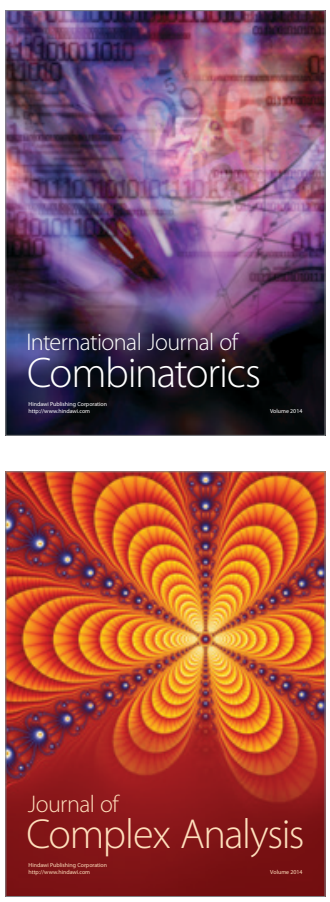

International Journal of

Mathematics and

Mathematical

Sciences
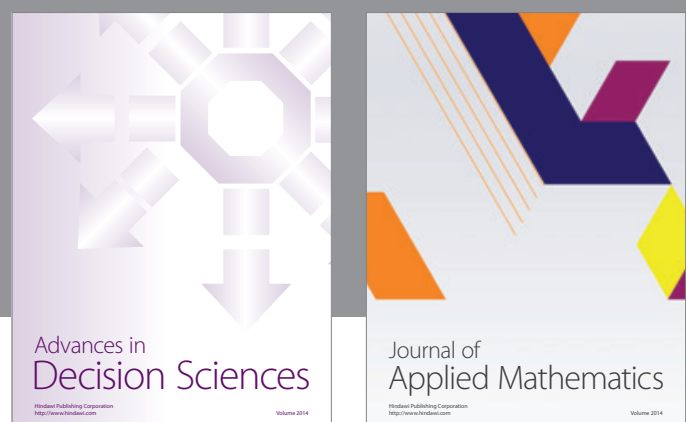

Journal of

Applied Mathematics
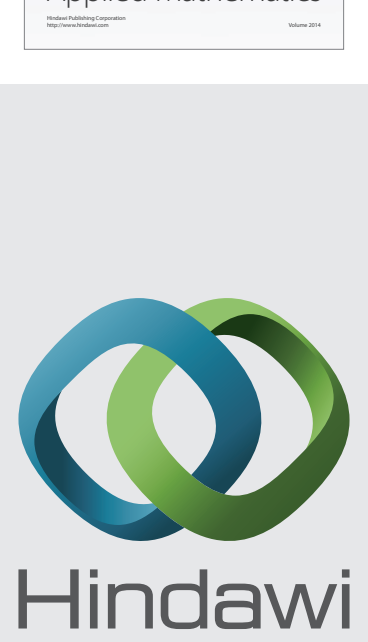

Submit your manuscripts at http://www.hindawi.com
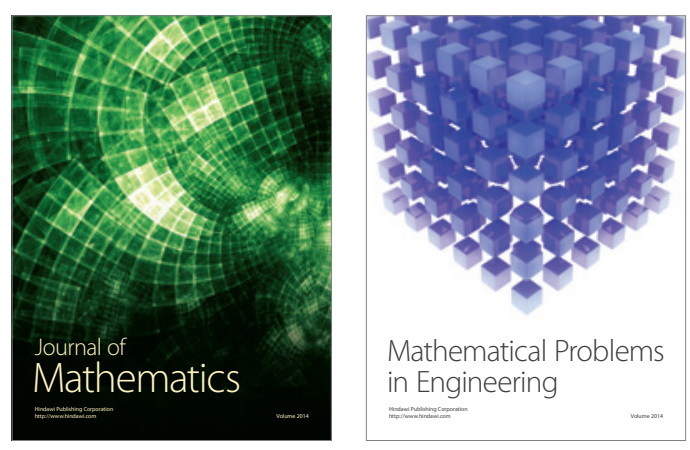

Mathematical Problems in Engineering
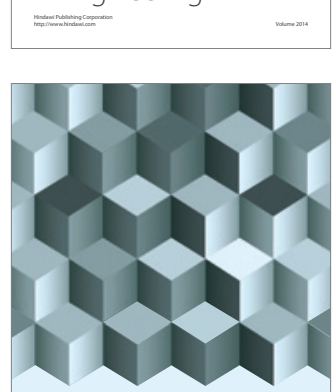

Journal of

Function Spaces
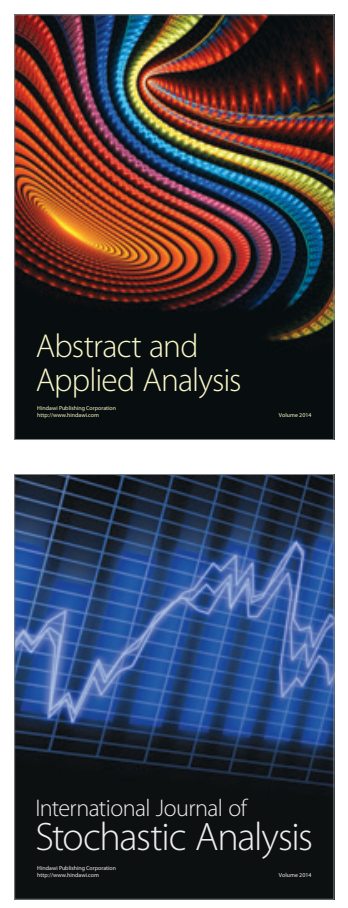

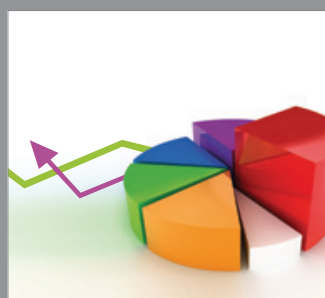

ournal of

Probability and Statistics

Promensencen
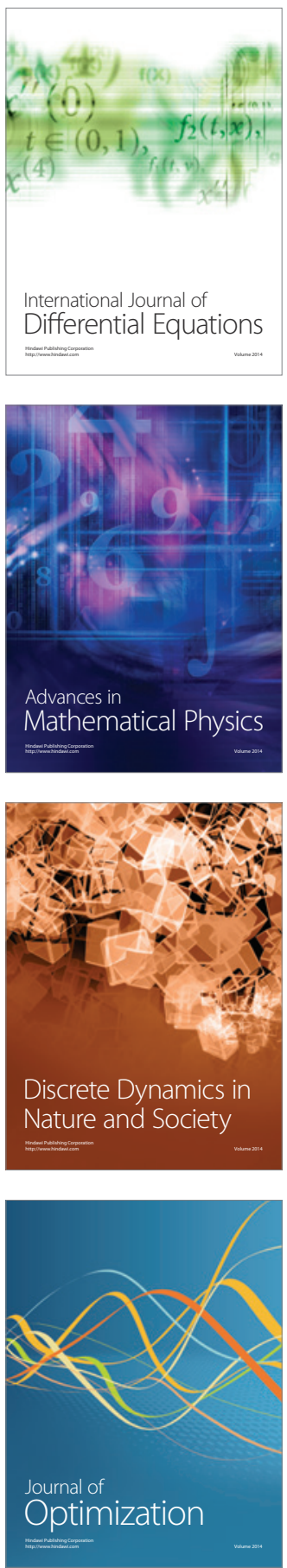